\title{
1 Baculovirus-vectored precision delivery of large DNA cargoes in human genomes
}

2

3 Francesco Aulicino ${ }^{1 *}$, Martin Pelosse ${ }^{1 \#}$, Christine Toelzer $^{1}$, Julien Capin ${ }^{1}$, Parisa Meysami ${ }^{1}$,

4 Mark Simon Dillingham ${ }^{1}$, Christiane Schaffitzel $^{1}$ and Imre Berger ${ }^{1,2 *}$

5

$6{ }^{1}$ BrisSynBio Bristol Synthetic Biology Centre, Biomedical Sciences, School of Biochemistry,

71 Tankard's Close, University of Bristol, Bristol BS8 1TD, United Kingdom

$8{ }^{2}$ Max Planck Bristol Centre for Minimal Biology, School of Chemistry, University of Bristol,

$9 \quad$ Clantock's Close, Bristol BS8 1TS, United Kingdom

10

11

12

13 " Current address: Laboratoire Chimie et Biologie des Métaux CEA Grenoble, 17 Avenue des

14 Martyrs 38054 Grenoble CEDEX 09 France

15

16 * Correspondence to francesco.aulicino@bristol.ac.uk and imre.berger@bristol.ac.uk 


\section{Abstract:}

19 Precise gene editing and genome engineering by CRISPR technology requires simultaneous

20 delivery of multiple DNA-encoded components into living cells rapidly exceeding the cargo

21 capacity of currently utilized viral vector systems. Here we exploit the unmatched heterologous

22 DNA cargo capacity of baculovirus to resolve this bottleneck. We implement hybrid DNA

23 techniques (MultiMate) for rapid and error-free assembly of currently up to 25 functional DNA

24 modules in a single baculoviral vector enabling CRISPR-based genome engineering. Utilizing

25 homology-independent targeted integration (HITI), we achieve up to $30 \%$ correct genome

26 interventions in human cells, including precision docking of large DNA payloads in the $A C T B$

27 locus. We demonstrate baculovirus-vectored delivery of prime-editing toolkits for seamless

28 DNA search-and-replace interventions achieving, with a single vector, highly efficient

29 cleavage-free trinucleotide insertion in the $H E K 3$ locus without any detectable indels. Our

30 approach thus unlocks a wide range of editing and engineering applications in human cell

31 genomes. 
Main

34 CRISPR/Cas represents a game-changing gene editing tool ${ }^{1}$. A programmable DNA nuclease (Cas9) is precisely guided to a specific DNA locus by means of a short single guide RNA (sgRNA) to elicit double strand DNA breaks (DSBs) subsequently repaired through nonhomologous end-joining (NHEJ) introducing small insertions-deletions (indels), giving rise to frameshift mutations and functional gene knock-outs $(\mathrm{KOs})^{1}$. Unpredictable indels that likewise occur are however undesirable in the context of therapeutic gene editing. Precise gene editing in contrast is typically achieved through homology directed repair (HDR) by providing a DNA template flanked by homology arms of variable length ${ }^{1,2}$, resulting in precise gene correction or knock-in (KIs) ${ }^{1}$. HDR activity however is intrinsically low and mostly restricted to $\mathrm{S} / \mathrm{G} 2$ cell cycle phases $^{3-5}$, reducing the efficiency of the desired gene editing outcome.

Significant effort is being made to increase the efficacy of CRISPR-HDR by means of smallmolecule NHEJ inhibitors ${ }^{6}$, cell cycle stabilised Cas9 variants $^{7,8}$ and other strategies ${ }^{9,10}$, however, gene editing efficiency in vivo has remained low.

More recently, homology-independent targeted integration (HITI), was shown to efficiently induce base pair precise KIs in both dividing and non-dividing cells ${ }^{11-13}$ by exploiting NHEJ and Cas9 cleaved donors, with exciting potential for gene editing applications in vivo ${ }^{11,14}$. Moreover, single base substitutions can also be achieved by using base editors (BEs) involving catalytically impaired Cas9 variants fused to cytosine or adenine deaminase ${ }^{15,16}$ and, more recently, prime editors (PEs) using Cas9 nickase fused to reverse transcriptase, achieving genomic interventions with little to no indels and reducing the risks associated with

$54 \mathrm{DSBs}^{17}$. BE, PE and HITI share in common a reliance on multiple functional DNA and protein elements that must be simultaneously delivered into target cells. This limits their applicability, in particular for future bona fide therapeutic interventions that necessitate a systemic approach and where co-transfection of plasmids and proteins, and likewise co-infection of viral vectors, 
will be problematic or unfeasible. In summary, the large DNA cargo capacity required for implementing these next-generation genomic interventions in vivo stands at odds with the limited cargo capacity of available technology, including the currently dominating adenoassociated virus (AAVs) and lentivirus (LVs) vector systems ${ }^{18}$. In marked contrast, baculoviral vectors (BVs) have a heterologous DNA cargo capacity far exceeding AAV and LV ${ }^{18-20}$. BVs, suitably pseudotyped to alter host cell tropism, are already widely used to transduce mammalian cells and living organisms ${ }^{19-22}$. We demonstrated in a proof of concept that BV is suitable for CRISPR-HDR mediated small $(\sim 0.7 \mathrm{~kb})$ DNA insertions with low efficacy $(\sim 5 \%)^{19,23}$, as would be expected for $\mathrm{HDR}^{3-5}$.

Here we unlock baculovirus as a vector of choice for next-generation genome intervention approaches, fully exploiting its unprecedented DNA cargo capacity and versatility. We implement and deploy state-of-the-art DNA assembly technology (MultiMate) for errorfree bottom-up assembly of multifunctional DNA circuitry comprising the DNA cargo to be inserted as well as all the DNA encoding all CRISPR modalities required to achieve highly efficient genome intervention in a baculovirus-vectored approach.

\section{Results}

We had previously developed methods to rapidly assemble functional DNA elements into multicomponent circuitry in baculoviral vectors ${ }^{24-26}$. Here, we optimized and fine-tuned our approach by incorporating time-tested MultiSite Gateway recombination modalities to assemble with ease currently up to 25 distinct DNA elements of various sizes (Fig.1a,

Extended Data Fig.1a-e, Supplementary Methods), importantly aiming to significantly reduce prokaryotic elements carried over into the baculovirus which can compromise vector

81 integrity during manufacturing ${ }^{27}$ (Extended Data Fig.1f). We validated the MultiMate system

82 by expressing the eight subunits of human chaperonin CCT/TRiC complex ${ }^{28}$ in insect cells 

cells (Fig.1b, Extended Data Fig.2a). MultiMate assembly yielded remarkably low error rates validating our approach (Extended Data Fig.2b). We deployed BVs harbouring 18 and 23.4 $\mathrm{kb}$ of MultiMate assembled functional DNA to efficiently transduce HEK293T, HeLa, H4 and SH-SY5Y leading to homogeneous expression and correct subcellular localization of all fluorescently labelled proteins (Fig.1b, Extended Data Fig.2c). Moreover, we created MultiMate-CellCycle $(9.1 \mathrm{~kb})$ as an improved cell cycle tracking tool implementing FUCCI reporters $^{29}$ as well as $\mathrm{H} 2 \mathrm{~B}-\mathrm{iRFP}$ expression for accurate transduction efficiency monitoring and cell cycle stage assessment (Fig.2c, Extended Data Fig.2d,e). MultiMate-CellCycle BVs efficiently transduced HEK293T, HeLa, H4 and SH-SY5Y cells highlighting differences in their respective cell cycle progressions (Extended Data Fig.2f) and enabling 12-hrs time lapse imaging on living HeLa cells, allowing cell cycle tracking also during FUCCI-unstained stages (M-G1 transition) (Fig1.c, Supplementary Video 1). These results comprehensively validate the MultiMate assembly platform enabling a wide range of baculovirus-vectored applications.

\section{Baculovirus-vectored homology independent targeted integration (HITI)}

To date, baculovirus-vectored gene editing approaches were confined to CRISPR-HDR of

102 small insert DNAs with low efficacy ${ }^{19,22,23}$. HITI toolkits using a viral vector required donor 103 and Cas9/sgRNA to be split between two AAVs due to their limited cargo capacity ${ }^{11}$, restricting successful gene editing to the fraction of co-infected cells. Moreover, manufacturing complete 'all-in-one' HITI vectors is not possible when viral packaging is performed in mammalian cells (typically HEK293T for AAV, LV), because simultaneous expression of Cas9 and sgRNA would inevitably excise the HITI donor, fatally compromising virus production. In marked 
contrast, BVs are manufactured in insect cells, where the mammalian promoters controlling

109 Cas9 and sgRNA expression are silent and all-in-one HITI construct packaging into the BV is

110 thus entirely feasible.

111 To minimise unpredictable indels and maximise correctly-edited alleles, we sought to

112 analyse and compare HDR and HITI-2 $\mathrm{c}^{11}$ strategies by targeting the intronic $\beta$-actin $(A C T B)$

113 locus, introducing a synthetic C-terminal exon fused to mCherry and a self-cleaving peptide

$114(\mathrm{~T} 2 \mathrm{~A})^{30}$ followed by a puromycin selection cassette (Fig.2a). We eliminated any possibility of

115 leaky expression by outfitting our DNAs with the Cas9 inhibitor AcrII4 ${ }^{31}$ under control of a

116 dual prokaryotic and baculoviral promoter (J23119-polH). An additional module expressing

117 eGFP under the constitutive CMV promoter was added to track transduction efficiency. Despite comparable transduction of HEK293T cells, Multimate-HITI-2c resulted in a 3- to 4-fold higher percentage of mCherry+ cells compared to Multimate-HDR (Fig.2b-c, Extended Data

Fig.3h). The BV backbone was rapidly diluted as we expected, while mCherry+ cells were stably maintained over time (Fig.2b-c) with absolute gene editing efficiencies reaching 5\% (HDR) and 20\% (HITI-2c) (Fig.2c). Notably, when compared to plasmid transfection, baculovirus-vectored delivery increased gene editing efficiency up to 4-fold regardless of the approach (Extended Data Fig.3a-c). To confirm correct gene editing, BV transduced cells were selected with puromycin and then expanded in the absence of selective pressure, demonstrating stably maintained mCherry expression in close to all (>98\%) cells (Fig.2d).

127 Successful editing was confirmed by PCR genotyping (Extended Data Fig.3e), the expected 128 mCherry subcellular localization (Fig.2e,Extended Data Fig.3f) and the predicted 129 ACTB::mCherry molecular weight (68 kDa) in western blot (Fig.2f, Extended Data Fig.3g).

130 MultiMate-HITI-2c ACTB outperformed HDR editing in all BV transduced cell lines

131 (Extended Data Fig.3h). For optimal efficacy, we prepared VSV-G pseudotyped BVs ${ }^{19}$ and 132 transduced HEK293T, HeLa, H4 and SH-SY5Y at different multiplicities of transduction 
133 (MOT) achieving higher transduction (up to 100\%) and editing efficiencies (up to 30\%)

134 depending on the cell line (Fig.2h, Extended Data Fig.3i). To our best knowledge this is the

135 first assembly and delivery platform to enable efficient homology independent targeted

136 integration in mammalian cells using a single all-in-one viral vector.

138 Safe-harbour integration of large DNA payloads

139 Precision docking of large multicomponent DNA circuitry in mammalian genomes is a

140 prerequisite for bona fide genome engineering, but remains an impeding challenge for currently

141 available viral delivery systems which are constrained by their intrinsic packaging limitations

142 (AAV: $\sim 4 \mathrm{~kb}$; LV: $\sim 8 \mathrm{~kb})^{18,20}$. We assessed the aptitude of our system for precision DNA

143 docking exploiting MultiMate-HITI-2c (Supplementary Methods). We used a new Cre

144 insertion site to generate a series of HITI-2c payloads ranging from $4.7 \mathrm{~kb}$ to $18 \mathrm{~kb}$ with

145 mTagBFP as a transduction efficiency reporter, resulting in all-in-one MultiMate plasmids of

146 up to $30 \mathrm{~kb}$ (Fig.3a). Transduction with EMBacY BV ${ }^{32}$ markedly outcompeted plasmid

147 transfection and editing in HEK293T (Extended Data Fig.4a-d). Upon puromycin selection,

148 cells remained $>98 \%$ mCherry+ (Extended Data Fig.4e) confirming precise 5'-end

149 integration. We observed silencing of the 3'-end fluorescent marker correlated with cargo size,

150 which we could fully restore by hygromycin selection (Fig.3b, Extended Data Fig.4f). We

151 confirmed correct integration by PCR genotyping and Sanger sequencing (Fig.3c,d). We

152 deployed MultiMate-HITI-2c 18K-CGH (30 kb) for baculovirus-vectored delivery with VSV-

153 G pseudotyped BV achieving 100\% transduction efficiency in both HEK293T and SH-SY5Y

154 giving rise to $20 \%$ and $30 \%$ absolute genomic insertion efficiency, respectively (Fig.3e). Of

155 note, mCherry expression remained constant over time in the absence of any selective pressure

156 while silencing of 3' eGFP expression (Fig.3f,g) was again promptly restored by

157 puromycin/hygromycin selection, remaining stable thereafter (Fig.3h). Our results 
demonstrate that safe-harbour integration of extensive DNA payloads with base-pair precision can be achieved with high-efficiency using all-in-one MultiMate-HITI-2c BVs, setting the stage for future large synthetic gene regulatory network engineering in human genomes.

Highly efficient baculovirus-vectored search-and-replace gene editing

163 Base editors $(\mathrm{BEs})^{15,16}$ and prime editing $(\mathrm{PEs})^{17}$ are new additions to the CRISPR toolkit that could potentially correct up to $89 \%$ of the human disease-causing mutations in the absence of indels production. PE exploits the nickase Cas9-H840A fused to reverse transcriptase from MMLV (PE2) to make a ssDNA copy of the engineered PegRNA at the edited site, allowing for the generation of all possible point mutations, insertions (up to $44 \mathrm{bp}$ ) and deletions (up to $80 \mathrm{bp}$ ) in the absence of DNA cleavage ${ }^{17}$. We chose here to insert a CTT trinucleotide in the HEK3 locus using the prime editing enzyme PE2 ${ }^{17}$. PE2 coding sequence spans $6.3 \mathrm{~kb}$ (excluding promoter) encoding for a $240 \mathrm{kDa}$ protein that previously could be virally delivered only through multiple split-intein lentiviral vectors ${ }^{17}$ due to cargo limitation, but is entirely within the packaging size of BV. We therefore assembled MultiMate-PE2 comprising PE2, HEK3 PegRNA cassette, VSV-G (for pseudotyping), aeBlue chromoprotein ${ }^{33}$ (for visual readout of virus titer) and mTagBFP (for transduction tracking) (Fig4. a, Extended Data Fig.5a). MultiMate-PE2 plasmid transfection resulted in 13\% CTT insertion in HEK3 as

177 assessed by Sanger sequencing and deconvolution using ICE $^{34}$ (Extended Data Fig.5b,c).

178 MultiMate-PE2 BVs production could be easily monitored (Extended Data Fig.5d) with excellent transduction efficiencies in the cell lines tested (Fig4b,d and Extended Data Fig.5e).

180 Sanger sequencing on $H E K 3$ locus amplicons from unsorted cells showed correct CTT editing 181 with base-pair precision (Fig.4c) with correct editing contributions after deconvolution of 15$18245 \%$, depending on the cell line, and undetectable indels (Fig.4e). We increased the 
transduction titer and observed a dose-dependent effect boosting correct editing events (up to 45\%) again without any indels detected (Extended Data Fig.5f-i) unlocking PE to our baculovirus-vectored approach for future, conceivably multiplexed applications including therapeutic interventions.

\section{Discussion}

189 The limited packaging capacity of currently dominating viral vectors (AAV, LV) markedly

190 constrains precise genome editing interventions ${ }^{18,20}$. We demonstrated here the efficacy of our

191 baculovirus-vectored approach to overcome this limitation. We developed a rapid error-free

192 DNA assembly (MultiMate) to facilitate vector construction with a view to improve BV manufacturing. We implemented homology independent targeted integration (HITI) for precise DNA insertion achieving efficient $\mathrm{C}$-terminal tagging in the $A C T B$ locus, markedly integration with outstanding efficacy of large multicomponent DNA payloads. There is no

197 indication that we reached the cargo limit of baculovirus-vectored delivery. In fact, given the

198 wide variation in size of naturally occurring baculoviruses ${ }^{35}$ we expect that delivery of DNA cargos exceeding $100 \mathrm{~kb}$ will likely be feasible, enabling insertion of entire metabolic pathways and gene regulatory networks in safe-harbour sites or elsewhere in genomes, additionally incorporating DNA insulator elements ${ }^{36,37}$ to support sustained gene expression. Importantly,

202 we demonstrate the utility of our approach for seamless search-and-replace gene editing by 203 implementing recently developed prime editing (PE) technology ${ }^{17}$. Particularly PE, is considered safer when compared to HDR or HITI-2c, which both rely on DNA cleavage. Using 
cargoes in human genomes, and we anticipate baculovirus-vectored large-scale genome

interventions, even combining safe-harbour integration with concomitant, if needed

211 therapeutic approaches in the future.

\section{References}

2141 Ran, F. A. et al. Genome engineering using the CRISPR-Cas9 system. Nature $215 \quad$ Protocols 8, 2281 (2013).

2162 Shy, B. R., MacDougall, M. S., Clarke, R. \& Merrill, B. J. Co-incident insertion enables high efficiency genome engineering in mouse embryonic stem cells. Nucleic Acids Research 44, 7997-8010 (2016).

3 Lieber, M. R. The Mechanism of Double-Strand DNA Break Repair by the Nonhomologous DNA End-Joining Pathway. Annual Review of Biochemistry 79, 181211 (2010).

4 Saha, J., Wang, S.-Y. \& Davis, A. J. Examining DNA Double-Strand Break Repair in a Cell Cycle-Dependent Manner. Methods Enzymol 591, 97-118 (2017).

5 Orthwein, A. et al. A mechanism for the suppression of homologous recombination in G1 cells. Nature 528, 422-426 (2015).

$6 \mathrm{Hu}, \mathrm{Z}$. et al. Ligase IV inhibitor SCR7 enhances gene editing directed by CRISPRCas9 and ssODN in human cancer cells. Cell \& Bioscience 8, 12 (2018).

7 Gutschner, T., Haemmerle, M., Genovese, G., Draetta, Giulio F. \& Chin, L. Posttranslational Regulation of Cas9 during G1 Enhances Homology-Directed Repair. Cell Reports 14, 1555-1566 (2016).

8 Yang, D. et al. Enrichment of G2/M cell cycle phase in human pluripotent stem cells enhances HDR-mediated gene repair with customizable endonucleases. Scientific Reports 6, 21264 (2016).

9 Canny, M. D. et al. Inhibition of 53BP1 favors homology-dependent DNA repair and increases CRISPR-Cas9 genome-editing efficiency. Nature Biotechnology 36, 95-102 (2018).

10 Haapaniemi, E., Botla, S., Persson, J., Schmierer, B. \& Taipale, J. CRISPR-Cas9 genome editing induces a p53-mediated DNA damage response. Nature Medicine 24, 927-930 (2018).

11 Suzuki, K. et al. In vivo genome editing via CRISPR/Cas9 mediated homologyindependent targeted integration. Nature 540, 144 (2016).

12 Kelly, J. J. et al. A Safe Harbor-Targeted CRISPR/Cas9 Homology Independent Targeted Integration (HITI) System for Multi-Modality Reporter Gene-Based Cell Tracking. bioRxiv, 2020.2002.2010.942672 (2020).

13 Artegiani, B. et al. Fast and efficient generation of knock-in human organoids using homology-independent CRISPR-Cas9 precision genome editing. Nature Cell Biology 22, 321-331 (2020). tool for gene therapy. J Hum Genet 63, 157-164 (2018). 
25015 Komor, A. C., Kim, Y. B., Packer, M. S., Zuris, J. A. \& Liu, D. R. Programmable editing of a target base in genomic DNA without double-stranded DNA cleavage. Nature 533, 420-424 (2016).

254 Gaudelli, N. M. et al. Programmable base editing of $\mathrm{A} \bullet \mathrm{T}$ to $\mathrm{G} \bullet \mathrm{C}$ in genomic DNA without DNA cleavage. Nature 551, 464-471 (2017). Anzalone, A. V. et al. Search-and-replace genome editing without double-strand breaks or donor DNA. Nature 576, 149-157 (2019).

Sung, L.-Y. et al. Efficient gene delivery into cell lines and stem cells using baculovirus. Nature Protocols 9, 1882-1899 (2014)

19 Mansouri, M. et al. Highly efficient baculovirus-mediated multigene delivery in primary cells. Nature Communications 7, 11529 (2016).

20 Kalesnykas, G. et al. Comparative Study of Adeno-associated Virus, Adenovirus, Bacu lovirus and Lentivirus Vectors for Gene Therapy of the Eyes. Curr Gene Ther 17, 235247 (2017).

21 Kost, T. A., Condreay, J. P. \& Jarvis, D. L. Baculovirus as versatile vectors for protein expression in insect and mammalian cells. Nat Biotechnol 23, 567-575 (2005).

22 Hindriksen, S. et al. Baculoviral delivery of CRISPR/Cas9 facilitates efficient genome editing in human cells. PLoS One 12, e0179514 (2017).

23 Mansouri, M., Ehsaei, Z., Taylor, V. \& Berger, P. Baculovirus-based genome editing in primary cells. Plasmid 90, 5-9 (2017).

24 Bieniossek, C. et al. Automated unrestricted multigene recombineering for multiprotein complex production. Nat Methods 6, 447-450 (2009).

25 Sari, D. et al. The MultiBac Baculovirus/Insect Cell Expression Vector System for Producing Complex Protein Biologics. Adv Exp Med Biol 896, 199-215 (2016).

26 Nie, Y. et al. ACEMBL Tool-Kits for High-Throughput Multigene Delivery and Expression in Prokaryotic and Eukaryotic Hosts. Adv Exp Med Biol 896, 27-42 (2016).

27 Pijlman, G. P., de Vrij, J., van den End, F. J., Vlak, J. M. \& Martens, D. E. Evaluation of baculovirus expression vectors with enhanced stability in continuous cascaded insect-cell bioreactors. Biotechnol Bioeng 87, 743-753 (2004).

28 Yam, A. Y. et al. Defining the TRiC/CCT interactome links chaperonin function to stabilization of newly made proteins with complex topologies. Nat Struct Mol Biol 15, 1255-1262 (2008).

29 Bajar, B. T. et al. Fluorescent indicators for simultaneous reporting of all four cell cycle phases. Nature methods 13, 993-996 (2016).

30 Liu, Z. et al. Systematic comparison of $2 \mathrm{~A}$ peptides for cloning multi-genes in a polycistronic vector. Scientific Reports 7, 2193 (2017).

31 Rauch, B. J. et al. Inhibition of CRISPR-Cas9 with Bacteriophage Proteins. Cell 168, 150-158.e110 (2017).

32 Fitzgerald, D. J. et al. Protein complex expression by using multigene baculoviral vectors. Nat Methods 3, 1021-1032 (2006).

33 Liljeruhm, J. et al. Engineering a palette of eukaryotic chromoproteins for bacterial synthetic biology. Journal of Biological Engineering 12, 8 (2018).

34 Hsiau, T. et al. Inference of CRISPR Edits from Sanger Trace Data. bioRxiv, 251082 (2018).

35 Vijayachandran, L. S. et al. Gene gymnastics: Synthetic biology for baculovirus expression vector system engineering. Bioengineered 4, 279-287 (2013).

36 Alhaji, S. Y., Ngai, S. C. \& Abdullah, S. Silencing of transgene expression in mammalian cells by DNA methylation and histone modifications in gene therapy perspective. Biotechnology and Genetic Engineering Reviews 35, 1-25 (2019). 
37 Harraghy, N., Gaussin, A. \& Mermod, N. Sustained transgene expression using MAR elements. Curr Gene Ther 8, 353-366 (2008).

\section{Figure 1. MultiMate enables rapid modular assembly of multifunctional DNA circuitry}

for efficient baculovirus-vectored delivery in human cells. a, MultiMate assembly platform in a schematic view. Symbols are listed (left panel). attL/R flanked DNA from ENTR plasmid plasmids, maximally eliminating prokaryotic backbone DNA sequences. MultiMate plasmids

310 are integrated in BVs customized for efficient delivery in human cells (right panel). b,

311 Confocal live cell imaging of H4, HeLa, HEK293T and SH-SY5Y 48 hours after transduction expression and correct subcellular localization of H2B-iRFP (nucleus), GTS-mTagBFP

314 (Golgi), mAG- $\beta$-catenin (membrane and adherens junctions), EYFP-Tubulin (microtubules),

315 mTFP1-Actin (cytoskeleton), mito-mCherry (mitochondria) and CyOFP1-ER (endoplasmic reticulum). Scalebar, $20 \mu \mathrm{m}$. c, Twelve-hours confocal time-lapse imaging of live HeLa cells cells constitutively express H2B-iRFP for DNA imaging. mAG-hGeminin and mKO2-hCdt1 are stabilised in S/G2 and G1 cell cycle stages, respectively. Upper panel shows G1/S transition, lower panel shows a dividing cell (arrows indicate tracked cells). Scalebar, $10 \mu \mathrm{m}$. DNA elements, plasmid topology and cell cycle schematics are illustrated (upper panel). 
targeted integration (HITI-2c) elements within attL1/attR3 sites (triangles) and MultiMate-

HITI-2c ACTB all-in-one DNA circuitry comprising Cas9, HITI-2c donor, sgRNA cassette,

promoters ensures vector stability. $A C T B \mathrm{C}$-terminal exon is replaced with a synthetic exon,

tagged with mCherry::T2A::puromycin. b-c, HEK293T cells transduced with MultiMate-HDR

or MultiMate-HITI-2c BVs in the absence of puromycin selection, at three- and ten-days post-

transduction. b, Representative FACS plots and $\mathbf{c}$, histogram of flow-cytometry data. Mean \pm

s.d. of $\mathrm{n}=3$ independent biological replicates. $* * * \mathrm{P}<0.001$, Student's t-test. d-f, HEK293T

transduced with MultiMate HDR or MultiMate HITI-2c BVs after puromycin selection. d,

Representative flow-cytometry histograms; e, Widefield microscopy, Scalebar $=20 \mu \mathrm{m}$. f,

336 Western blot of total protein extracts. Anti- $\beta$-actin antibody was used in top panel with anti-

337 TUBULIN as loading control. g, Confocal images of HEK293T, HeLa, H4 and SH-SY5Y cells

MultiMate-HITI-2c VSV-G pseudotyped BVs, multiplicity of transduction (MOT) 1 and 10.

341 Transduction efficiency $=\%$ of eGFP + cells; absolute gene editing efficiency $=\%$ of Cherry + cells. Mean \pm s.d. of $n=3$ independent biological replicates.

344 Figure 3. Baculovirus-vectored safe-harbour homology-independent integration of large

DNA cargoes in human genomes. a, Safe-harbour HITI-2c strategy, HITI-2c payloads within

346 attL1/attR3 sites (triangles) and MultiMate-HITI-2c ACTB all-in-one plasmid carrying Cas9,

347 HITI-2c payload, sgRNA, AcrII4 and mTagBFP reporter. ACTB C-terminal exon is replaced

348 with a synthetic exon, tagged with T2A::mCherry::P2A::puromycin (5' integration marker),

349 DNA insert ranging from 4.7 to $18 \mathrm{~kb}$, and distinct 3' integration markers (CMV Hygromycin

$350(\mathrm{CH}), \mathrm{CMV}$ eGFP IRES Hygromycin $(\mathrm{CGH})$ or EF1a EYFP-Tubulin IRES Hygromycin 
351 (EYH)). b-c, HEK293T transduced with the indicated MultiMate-HITI-2c BVs after

352 puromycin and hygromycin selection. b, Confocal microscopy. Scalebar, $100 \mu \mathrm{m}$. c, PCR

353 genotyping. Oligonucleotide pairs (colour coded arrows) for each PCR are shown, their

354 approximative position is depicted in (a). d, Sanger sequencing of 5' and 3' genotyping PCRs

355 (indicated by $*$ in (c)) of HEK293T transduced with MultiMate-HITI-2c 18K-CGH BV. e,

356 Transduction efficiency (left histogram) and absolute gene editing efficiency (right histogram)

357 of HEK293T and SH-SY5Y 72 hours after transduction with MultiMate-HITI-2c 18K-CGH

358 VSV-G pseudotyped BV, derived from flow-cytometry data. Mean \pm s.d. of $n=3$ independent

359 biological replicates. f, Confocal microscopy pictures of HEK293T and SH-SY5Y 8 days after

360 transduction with MultiMate-HITI-2c 18K-CGH VSV-G pseudotyped BVs in the absence of

361 puromycin or hygromycin selection. g, Representative flow-cytometry plots of HEK29T at

362 three- or 24-days post transduction with MultiMate-HITI-2c 18K-CGH VSV-G pseudotyped

363 BV. h, Representative flow-cytometry plots of HEK29T transduced with MultiMate-HITI-2c

364 18K-CGH VSV-G pseudotyped BV after puromycin (left) and puromycin/hygromycin

365 selection (right).

Figure 4. Highly efficient and indels-free prime-editing by using MultiMate all-in-one BV.

a, MultiMate-PE2 for CTT insertion in the HEK3 locus in a schematic view. aeBlue is used

369 for tracking viral amplification and VSV-G for pseudotyping BV, respectively. mTagBFP

370 reports on transfection/transduction efficiency in human cells. b, Representative flow-

371 cytometry histograms of HeLa, RPE-1 hTERT, HEK293T and SH-SY5Y cells at 24 hours post

372 transduction with MultiMate-PE2 VSV-G pseudotyped BV. c, Representative genotype PCR

373 sanger sequencing of unsorted HeLa, RPE-1 hTERT, HEK293T and SH-SY5Y cells at 96

374 hours post transduction with MultiMate-PE2 VSV-G pseudotyped BV and their respective

375 controls (untransduced parental cell lines). d, Transduction efficiencies of HeLa, RPE-1 
hTERT, HEK293T and SH-SY5Y cells at 24 hours post transduction with MultiMate-PE2

377 VSV-G pseudotyped BV. Mean \pm s.d. of $\mathrm{n}=3$ independent biological replicates. e, Percentage

378 of correct editing (CTT insertion) in HeLa, RPE-1 hTERT, HEK293T and SH-SY5Y at 96

379 hours post transduction. Data are derived from sanger sequencing deconvolution (ICE), no

380 indels were detected in any of the conditions. Mean \pm s.d. of $n=3$ independent biological

381 replicates.

382

Methods

\section{Gibson assembly of DNA elements}

385 An extensive list of constructs maps and assembly strategies is provided in Supplementary

386 Table 1. ENTR and DEST vectors were generated using Gibson assembly (NEB Builder Hi-

387 Fi DNA assembly \#E2621S) following manufacturer's instructions. Fragments were mixed

388 with a backbone to insert ratio of 1:1 ( $>3$ fragments), 1:2 ( $<2$ fragments), $1: 5$ (fragments $<300$

389 bp) in $5 \mu$ l total volume and supplemented with 5 ul 2xNEB Builder Hi-Fi mix followed by

390 incubation at $50^{\circ} \mathrm{C}$ for 1 hour. $2 \mathrm{ul}$ of the assembly mix were transformed into homemade

391 electrocompetent Top 10 or $\mathrm{Pir}^{+}$E. Coli. followed by recovery at $37^{\circ} \mathrm{C}$ while shaking for 1 hour

392 and plating on LB/agar plates with the appropriate antibiotics. All the precursors vectors

393 generated in this study were assembled using Gibson assembly of synthetic DNA fragments,

394 oligonucleotides, digested vectors or PCRs amplified with Herculase II fusion (Agilent\#

395 600675). A portion of pENTR-D-TOPO (Invitrogen) was used as backbone for all the pMMK

396 ENTR vectors. attL/R sites on pMMK ENTR vectors were obtained by overlap extent PCRs

397 of long oligonucelotides. attR1-Ccdb-Chlo-attR2 cassettes for generating pMMACE DEST

398 were PCR amplified from pInducer- $20^{38}$. pMMDS DEST was generated by replacing the

399 Chloramphenicol cassette with Ori ${ }^{\mathrm{ColE} 1}$ PCR amplified from pACEMam1 ${ }^{19}$. H2B-iRFP was

400 PCR amplified from pCAG-H2BtdiRFP-IP ${ }^{39}$, EYFP-Tubulin and mTFP1-Actin were 
amplified from 5-colours MultiBac vectors ${ }^{19}$. pMDK $7 \mathrm{~kb}$ and $12 \mathrm{~kb}$ were generated by cloning

402 promoterless and ATG-less portions of SMG1(NM_015092.5) CDS. Mito-mCherry was

404 from 5-colours MultiBac vectors ${ }^{19}$. CyOFP $1^{41}$ fused to the endoplasmic reticulum targeting 405 sequence (ER) was synthesised by Twist Bioscience. mAG $\beta$-catenin was obtained through 406 Gibson assembly of PCR amplified mAG from pL-EF1a mAG-hGeminin ${ }^{42}$, and $\beta$-catenin from 407 pL-EF1a $\beta$-catenin SV40 Puro ${ }^{43}$. Golgi targeting sequence (GTS) mTaBFP was synthesised by

408 Twist Bioscience. polH or p10 cassettes were amplified from MultiBac vectors ${ }^{32,44}$, CCT 409 isoforms were PCR amplified from synthetic vectors from GenScript. SpCas9 was PCR 410 amplified from $\mathrm{px} 459^{1}$, sgRNAs cloning was performed by overlap extent PCRs of hU6 and 411 scaffold fragments amplified from $\mathrm{px} 459^{1}$. HDR and HITI-2c templates were amplified by

412 Gibson assembly of genomic DNA fragments, mCherry was amplified from 7TGC ${ }^{40}$, T2A Puro

413 from $\mathrm{px} 459^{1}$. sgRNAs target in the HITI-2c donors were included as overlapping ends between 414 fragments. polH/J23119 AcrII4 ${ }^{31}$ was synthesised by Twist Bioscience. In the HITI-2c 415 payloads for safe-harbour integration, mCherry T2A Puro was replaced with T2A mCherry 416 P2A Puro. P2A was generated by overlap extent PCR of long oligonucleotides. The loxP site 417 was PCR amplified from pACEBac1 ${ }^{32,44}$, Hygromycin, IRES and eGFP were PCR-amplified 418 from p1494 vectors ${ }^{45}$. PE2 was obtained by restriction digestion from pCMV PE2 ${ }^{17}$ and the 419 HEK3 PegRNA was PCR amplified from pU6-Sp-pegRNA-HEK3_CTT_ins ${ }^{17}$. aeBlue ${ }^{33}$ was 420 synthesised by Twist Bioscience and VSVG-G was amplified from pMD2.G (Didier Trono lab, 421 Addgene plasmid \# 12259).

\section{LR recombination}

424 LR recombination was carried out using LR Clonase II (Thermo Fisher \#11791020) or LR 425 Clonase II plus (Thermo Fisher \#12538120). Although LR Clonase II plus is specifically 
designed for MultiSite Gateway recombination, LR Clonase II worked with comparable efficiency in our hands. LR reactions were carried out following manufacturer's instructions. (Thermo Fisher \#12090015). 1 ul of each diluted vector was added to a $0.2 \mathrm{ml}$ PCR tube with hours. The next day the reaction was terminated by addition of $1 \mathrm{ul}$ proteinase $\mathrm{K}$ (provided with LR Clonase II enzymes) and incubation at $37^{\circ} \mathrm{C}$ for 10 minutes. 2-3 ul were transformed into homemade electrocompetent Top 10 or $\mathrm{Pir}^{+}$E.Coli, followed by 2 hours recovery at $37^{\circ} \mathrm{C}$ and plating on LB/agar plates with the appropriate antibiotics. LR recombination products were predicted using $\mathrm{APE}^{46}$ with custom recombination reactions. To quickly load MultiMate LR reaction prototype in APE, the following code can be copied and used in Tools/Recombination

MultiMate LR Reaction for APE:

\{ApE recombination reaction:\} \{MultiMate LR Reaction (1-3-4-5-2)\} \{\{\{pMMK ENTR 1$\}$ \{pMMK ENTR 2\} \{pMMK ENTR 3\} \{pMMK ENTR 4\} DEST\} \{attB1 0 attB3 0 attB4 0 attB5 0 attB2 1\}\}

440 When imported as GenBank or Fasta files, the MultiMate LR reaction in APE will automatically recognize pMMK ENTR1-4 vectors and one DEST donors when launched through Tools/Recombination tools. For manual prediction of MultiMate assembly products, a list of the attL/R sequences and their attB products is provided in Supplementary Table 3.

\section{Cre-mediated recombination of DNA elements}

446 One acceptor and one or multiple donor vectors were assembled using Cre-mediated

447 recombination as previously described ${ }^{26,32,47}$. One acceptor and one or more donors were mixed 448 with a ratio of $1: 1.1$ in distilled $\mathrm{H} 2 \mathrm{O}$ with $0.5 \mathrm{ul}$ (7.5 U) of CRE recombinase (NEB \# M0298M) 
H2O. 500-1000 ng of total DNA were used for each reaction. Cre-reactions were incubated for

4511 hour at $37^{\circ} \mathrm{C}$, followed by heat inactivation at $70^{\circ} \mathrm{C}$ for 10 minutes. $2-3$ ul were transformed

452 into homemade electrocompetent Top10 E.Coli, followed by 2 hours recovery at $37^{\circ} \mathrm{C}$ and

453 plating on LB/agar plates with the appropriate antibiotics. Cre-recombination products were

454 predicted using Cre-ACEMBLER Vers. $2.0{ }^{47}$.

Cell culture methods

457 Human cells (HEK293T, HeLa, H4, RPE-1 hTERT and SH-SY5Y) were purchased from 458 ATCC and propagated as adherent cultures in 60 or $100 \mathrm{~cm}$ dishes in a humidified incubator $459\left(37^{\circ} \mathrm{C}, 5 \% \mathrm{CO} 2\right)$. For passaging cells were washed with phosphate saline buffer (DPBS, Gibco 460 \# 14190144), detached using 0.25\% Trypsin (Thermo Fisher \#25200056) followed by a brief incubation at $37^{\circ} \mathrm{C}$, centrifuged at $300 \mathrm{x}$ RCF and resuspended in fresh media in a new plate at the desired concentration. Suspension cultures of Sf21 insect cells were grown in $125 \mathrm{ml}$ or $250 \mathrm{ml}$ polycarbonate Erlenmeyer flasks with vent cap (CORNING, \#431143, \#431144) at $27^{\circ} \mathrm{C}$ in a shaking incubator. Sf 21 were split every $2 / 3$ days and maintained at concentrations between $0.5-2 \times 10^{6}$ cells $/ \mathrm{ml}$. Origin and media formulation recipe for each cell line is reported in Supplementary Table 4.

467 For transfection in HEK293T, 2x105 cells/well were seeded in multi-24 wells. Transfections were carried out using Polyfect (QIAGEN \#301105), following manufacturer's instructions.

469 Briefly $500 \mathrm{ng}$ of DNA were resuspended in 25 ul of Optimem (Gibco \#31985062), supplemented with 5 ul Polyfect and incubated for 15 minutes at room temperature.

471 Transfection mix was resuspended with $100 \mathrm{ul}$ of complete media and added dropwise to each 472 well. Cells were cultured for at least 48 hours before assessing the phenotype (e.g. fluorescence markers expression). 


\section{Baculovirus vector amplification}

476 Assembled MultiMate vectors were shuttled on baculovirus genomes (bacmids) propagated in

477 E.Coli using Tn7 transposition. 200-1000 ng of MultiMate vector were transformed in

478 chemically competent DH10-MultiBacMam-VSV-G ${ }^{19}$, DH10-EMBacY ${ }^{32}$ or commercial

479 DH10Bac (ThermoFisher \# 10361012) as previously described ${ }^{32}$. Bacteria were streaked on

480 LB/Agar plates with Gentamycin, Kanamycin, Tetracyclin, IPTG and Bluo-Gal and incubated

481 for 24-hours for blue-white screening. White colonies were picked and grown overnight in 3

$482 \mathrm{ml}$ of LB supplemented with Gentamycin/Kanamycin to extract bacmid DNA through alkaline

483 lysis/ethanol precipitation as previously described ${ }^{32,44}$.

484 For transfection in insect cells, $0.8-1 \times 10^{6} \mathrm{Sf} 21$ cells/well were seeded on multi- 6 well plates in

$4853 \mathrm{ml}$ of Sf-900 II media. $10 \mathrm{ul}$ of purified bacmid were resuspended in $130 \mathrm{ul} \mathrm{Sf-900} \mathrm{II} \mathrm{media}$

486 with 10 ul X-treme XP transfection reagent (Roche \# 06366236001) and incubated at room

487 temperature for 15 minutes. The entire transfection mix was added dropwise to a single well

488 and cells were incubated at $27^{\circ} \mathrm{C}$ in a static incubator. $\mathrm{V}_{0}$ viral stocks were harvested collecting

489 the supernatant of transfected cells $72-96$ hours post transfection as previously described ${ }^{19,32,47}$.

$490 \quad 1-3 \mathrm{ml}$ of $\mathrm{V}_{0}$ viral stocks was added to $10 \mathrm{ml}$ of fresh $\mathrm{Sf} 21$ cells at $0.8 \times 10^{6}$ cells $/ \mathrm{ml}$. Cells were

491 cultured in $50 \mathrm{ml}$ Falcon tubes while shaking at $27^{\circ} \mathrm{C}$ and counted every day to monitor cell

492 proliferation and size using Luna cell-counter (LogosBio). Successfully infected cells

493 displayed arrested proliferation and increased average cell size (13-14 $\mu \mathrm{m}$ control, 16-20 $\mu \mathrm{m}$

494 infected). $\mathrm{V}_{1}$ viral harvest were collected as previously described 2 days after proliferation

495 arrest $(\mathrm{DPA}+24)^{19,32,47}$ by centrifugation at $4500 \mathrm{x}$ rcf. $500 \mu \mathrm{l} / 1 \mathrm{ml}$ of $\mathrm{V}_{1}$ viral stocks was added

496 to $50 \mathrm{ml}$ of fresh $\mathrm{Sf} 21$ cells at $0.8 \times 10^{6}$ cells $/ \mathrm{ml}$ in $125 \mathrm{ml}$ Erlenmeyer flasks and cells were

497 cultured at $27^{\circ} \mathrm{C}$ in a shaking incubator. $\mathrm{V}_{2}$ viral harvests were collected by centrifugation at $4984500 \mathrm{x}$ rcf, and concentrated 20 times by high-speed centrifugation at $11000 \mathrm{x}$ rcf, followed by 

storage at $-80^{\circ} \mathrm{C}$.

501

\section{Baculovirus vector titration and transduction}

503 For BV expressing fluorescent markers in human cells, titration was performed as previously 504 described $^{48}$. HEK293T were used to determine viral titers. Briefly $5 \times 10^{5}$ cells $/$ well were seeded in multi-48 wells plates in $200 \mathrm{ul}$ of complete media. Concentrated virus was serially diluted in DPBS and $50 \mu 1$ were dispensed to each well.

507 Spinoculation $\left(30^{\prime}\right.$ at $600 \mathrm{x}$ rcf at $27^{\circ} \mathrm{C}$ ) was used to enhance transduction as previously 508 reported $^{48} .24$ hours after transduction, cells were analysed using flow-cytometry to determine 509 the percentage of transduced cells. TU/ml values from dilutions giving less than $20 \%$ transduction efficiencies were averaged to estimate the titer as previously described ${ }^{49}$ and using

511 Supplementary Equation 1. For experiments in which different viral titers were used,

512 multiplicity of transduction (MOT) was calculated as TU/Cn.

\section{Confocal and widefield imaging}

515 Confocal images were acquired using a Leica Sp8 equipped with 405, 458, 476, 488, 496, 514, $516561,594,633 \mathrm{~nm}$ laser lines and $37^{\circ} \mathrm{C}$ stage. For time lapse confocal experiments on living 517 cells the stage was supplemented with 5\% CO2. For higher magnification, cells were plated on 518 Lab-Tek borosilicate multi-8 wells (Thermo Fisher \# 155411). For experiments in which

519 transduced cells expressed more than 3 different fluorochromes, laser intensity and detection 520 filters were adjusted to reduce spectral overlap using individual fluorescence controls 521 transfections. Widefield and phase contrast images were acquired using a Leica DMI6000 522 equipped with excitation/emission filters optimised for DAPI, GFP, Rhodamine, Texas Red 523 and Far red. 


\section{Flow cytometry analysis}

526 For flow-cytometry analysis cells were trypsinised and resuspended in complete media supplemented with $3 \mu \mathrm{M}$ DRAQ7 (Abcam \#ab109202) to counterstain dead cells. Cells were analysed on a BD Fortessa, fluorochromes were detected as follow: eGFP and EYFP (FITCA), mCherry (PECF594-A), mTagBFP (BV421-A), DRAQ7 (AlexaFluor700-A). SSC-A and FSC-A were used to discriminate single cells and cell populations by size. FlowJo X was used to analyse FCS files. All data represented are percentages of live single cells (DRAQ7-).

PCR genotyping, Sanger sequencing and deconvolution

534 Genomic DNA was extracted with QIAamp DNA Mini Kit (QIAGEN \# 51306) following manufacturer's instruction. A list of the predicted gene editing outcome sequences and genotyping oligos is provided in Supplementary Table 2. PCRs were performed using

KAPA2G Fast Genotyping mix (SigmaAldrich \# KK5621) following manufacturer's instruction. Amplicons were run on $0.8 \%$ agarose gels, purified using QIAquick Gel Extraction Kit (QIAGEN \# 28706) and eluted in distilled ddH2O. For Sanger sequencing 15 ul of purified PCR at 5-10 ng/ $\mu 1$ were mixed with $2 \mu 1$ of diluted $(10 \mu \mathrm{M})$ sequencing primer and sent to an cells were fed into $\mathrm{ICE}^{34}$ from Synthego for sequence deconvolution and indels/knock-in estimation.

Western blot analysis

546 Total protein extracts from HEK293T were obtained by lysing the cells with ice-cold RIPA

547 Buffer (Thermo Fisher \# 89901) supplemented with protease inhibitors (Thermo Fisher \# $548 \quad 78429)$ for $30^{\prime}$ on ice. Insoluble material was pelleted by centrifugation at $16000 \mathrm{x}$ rcf at $4^{\circ} \mathrm{C}$ 
549 for 5 minutes. Total protein extracts from insect cells were obtained as previously described ${ }^{32}$.

550 Proteins concentration were determined using Nanodrop. $10 \mu \mathrm{g}$ protein/sample were stained

551 with Laemmli buffer, boiled at $95^{\circ} \mathrm{C}$ for 5 minutes, separated using pre-cast NuPage $4-12 \%$

552 Bis-Tris SDS-Gels (Thermo Fisher \# NP0321BOX) and transferred to PVDF membranes using

553 iBlot. Membranes were blocked with 5\% non-fat dry milk in T-TBS (50 mM Tris-Cl, pH 7.6,

$554150 \mathrm{mM} \mathrm{NaCl}, 0.5 \%$ Tween) for 1 hour at room temperature. Membranes were incubated with 555 primary antibodies diluted 1:1000 in T-TBS 5\% milk overnight at $4{ }^{\circ} \mathrm{C}$ while rocking, followed

556 by 2 T-TBS washes and incubation with HRP-conjugated secondary antibody diluted 1:2000

557 in T-TBS 5\% milk for 1 hour at room temperature. Membranes were washed again with T-TBS

558 and developed using Pierce ECL reagents (Thermo Fisher \# 34579) following manufacturer's

559 instructions. Finally, membranes were imaged using MyECL Imager.

560 A list of the primary and secondary antibodies used is provided in Supplementary Table 5.

\section{CCT/TriC complex purification}

563 Recombinant MultiMate-CCT BV was produced as previously described ${ }^{32}$ and used to infect

564 Sf21 insect cells at a cell density of $1.0 \times 10^{6} / \mathrm{mL}$ in Sf-900 II medium. Cells were harvested 72-

$56596 \mathrm{~h}$ after proliferation arrest by centrifugation at $1,000 \mathrm{~g}$ for 15 minutes. Cell pellets were resuspended in lysis buffer $(50 \mathrm{mM}$ HEPES-NaOH, $200 \mathrm{mM} \mathrm{KCl}, 10 \mathrm{mM}$ Imidazole, $20 \%$

567 Glycerol, pH 7.5, supplemented with EDTA-free protease inhibitor (Sigma-Aldrich) and

568 Benzonase (Sigma-Aldrich)) and lysed by short sonication. The lysate was cleared by

569 centrifugation at $18,000 \mathrm{rpm}, 4^{\circ} \mathrm{C}$, in a F21-8x50y rotor (Thermo Fisher Scientific) for 60

570 minutes. The supernatant was loaded on a TALON column (Generon), equilibrated in TALON

571 A buffer (50 mM HEPES-NaOH, 200 mM KCl, 10 mM Imidazole, $20 \%$ Glycerol, pH 7.5)

572 with a peristaltic pump. The column was washed with ten column volumes (CV) of TALON A

573 buffer before eluting the bound protein complex with a step gradient of TALON B buffer (50 
mM HEPES-NaOH, $200 \mathrm{mM} \mathrm{KCl,} 250$ mM Imidazole, $20 \%$ Glycerol, $\mathrm{pH}$ 7.5). The CCT

(GE Healthcare) and eluted with a $1 \mathrm{M} \mathrm{KCl}$ gradient in Heparin A buffer. Fully formed complexes and disassembled subunits were separated on a Superose 6 10/300 column (GE Healthcare) equilibrated in SEC buffer (20 mM HEPES-NaOH, $200 \mathrm{mM} \mathrm{KCl,} \mathrm{pH} \mathrm{7.5,} 1 \mathrm{mM}$ DTT, $10 \%$ Glycerol). Peak fractions were pooled and concentrated and the purity of the CCT complex was analyzed by SDS-PAGE.

\section{Electron microscopy}

584 For electron microscopy, copper grids with carbon coating (300 mesh, Electron Microscopy Sciences) were glow discharged for $10 \mathrm{~s}$, and $5 \mu \mathrm{L}$ of purified CCT was placed on the grids for $1 \mathrm{~min}$. Afterwards the grid was washed for $15 \mathrm{~s}$ and floated onto a drop of filtered $3 \%$ uranyl acetate for $1 \mathrm{~min}$. Excess solution on the grids was blotted off using filter paper between each step. Grids were visualized under a FEI Tecnai 20 transmission electron microscope (TEM), and digital micrographs were taken using a FEI Eagle 4Kx4K CCD camera. Particle picking and processing was performed using RELION $2^{50,51}, 2 \mathrm{D}$ class averages were generated without applying symmetry or reference models.

\section{Data availability statement}

594 All plasmid sequences are provided in Supplementary Table 1. MultiMate-CellCycle, MultiMate-Rainbow and MultiMate-HITI-2c ACTB reagents will be made available for

597 request. 


\section{Methods References}

600

601

38 Meerbrey, K. L. et al. The pINDUCER lentiviral toolkit for inducible RNA interference

602

603 in vitro and in vivo. Proceedings of the National Academy of Sciences 108, 3665 (2011).

604

605

606

607

608

609

610

611

612

613

614

615

616

617

618

619 Miyanari, Y., Ziegler-Birling, C. \& Torres-Padilla, M.-E. Live visualization of chromatin dynamics with fluorescent TALEs. Nature Structural \& Molecular Biology 20, 1321-1324 (2013).

40 Fuerer, C. \& Nusse, R. Lentiviral Vectors to Probe and Manipulate the Wnt Signaling Pathway. PLOS ONE 5, e9370 (2010).

41 Chu, J. et al. A bright cyan-excitable orange fluorescent protein facilitates dualemission microscopy and enhances bioluminescence imaging in vivo. Nature Biotechnology 34, 760 (2016).

42 De Jaime-Soguero, A. et al. Wnt/Tcf1 pathway restricts embryonic stem cell cycle through activation of the Ink4/Arf locus. PLOS Genetics 13, e1006682 (2017).

43 Aulicino, F. et al. Canonical Wnt pathway controls mESCs self-renewal through inhibition of spontaneous differentiation via $\beta$-catenin/TCF/LEF functions. bioRxiv, 661777 (2019).

44 Berger, I., Fitzgerald, D. J. \& Richmond, T. J. Baculovirus expression system for heterologous multiprotein complexes. Nat Biotechnol 22, 1583-1587 (2004).

45 Aulicino, F., Theka, I., Ombrato, L., Lluis, F. \& Cosma, M. P. Temporal perturbation of the Wnt signaling pathway in the control of cell reprogramming is modulated by

620

621

622

623

624

625

626

627

628

629

630

631

632 TCF1. Stem Cell Reports 2, 707-720 (2014).

46 Davis, M. W. ApE - A plasmid editor https://jorgensen.biology.utah.edu/wayned/ape/.

47 Becke, C., Haffke, M. \& Berger, I. Cre-ACEMBLER Software User Manual. (2012).

48 Cheng, T. et al. [Improving baculovirus transduction of mammalian cells by spinoculation]. Sheng Wu Gong Cheng Xue Bao 23, 546-551 (2007).

49 Lo, W.-H., Chen, C.-Y., Yeh, C.-N., Lin, C.-Y. \& Hu, Y.-C. Rapid baculovirus titration based on regulatable green fluorescent protein expression in mammalian cells. Enzyme and Microbial Technology 48, 13-18 (2011).

50 Kimanius, D., Forsberg, B. O., Scheres, S. H. \& Lindahl, E. Accelerated cryo-EM structure determination with parallelisation using GPUs in RELION-2. Elife 5 (2016).

51 Scheres, S. H. W. RELION: Implementation of a Bayesian approach to cryo-EM structure determination. Journal of Structural Biology 180, 519-530 (2012).

\section{Acknowledgement}

636 We thank all members of the Berger, Schaffitzel and Dillingham teams and our academic and 637 industrial collaborators for their contributions. We thank Daniel Fitzgerald (Geneva Biotech) 638 for helpful discussions. We acknowledge generous support from GE Healthcare through a

639 Discovery Research Grant (to I.B.), from BrisSynBio, a BBSRC/EPSRC Research Centre for 
641 Bristol Centre for Minimal Biology. I.B. is Investigator of the European Research Council

642 (ERC Advanced Grant DNA-DOCK, Project Nr. 834631).

643

\section{Author contributions}

645 I.B. and F.A. conceived and designed the study with input from C.S. and M.S.D.. F.A. 646 performed experiments and analysed results. F.A. and M.P. designed, implemented and 647 validated DNA assembly protocols. C.T. performed protein purification and EM. J.C and P.M. 648 produced and provided reagents. M.S.D., C.S. and I.B. supervised and guided the research. 649 F.A. and I.B. wrote the manuscript with input from all authors.

650

\section{Competing interest statement}

652 I.B. declares shareholding in Geneva Biotech Sàrl and is inventor on patents and patent applications covering DNA methods and baculovirus reagents licensed to Geneva Biotech Sàrl.

Additional Information

656 Supplementary Information contains: Supplementary Methods detailing MultiMate DNA assembly and MultiMate-HITI-2c safe harbour assembly; Supplementary Tables providing sequences, gene editing outcomes and genotyping oligonucleotides sequences, cell lines and antibodies; Supplementary Equation and Supplementary Video.

660

\section{Extended Data Figure Legends}

662 Extended Data Figure 1. MultiMate assembly protocol and validation. a, DNA elements

663 are pasted into four distinct ENTR plasmid modules in between attL/R recombination sites

664 (coloured triangles). b, One DEST module and four ENTR modules are assembled by LR

665 recombination in vitro. DEST plasmids are adapted from MultiBac Acceptor/Donor suite ${ }^{25,32}$. 
666

667

668

669

670

671

672

673

674

675

676

677

678

679

680

681

682

683

684

685

686

688

689

690

In pMMACE DEST, the homing cassette is attR1-Ccdb-Chlo ${ }^{\mathrm{R}}$-attR2, in pMMDS DEST attR1Ccdb-Ori ${ }^{\mathrm{ColE} 1}$-attR2. $\mathbf{c}$, After LR reaction, $\mathrm{pMMACE}$ DEST and pMMDS DEST are fused by Cre-recombination. d, MultiBac donor plasmids and pMMD DEST vectors can be iteratively added by Cre recombination, barcoded by resistance makers ${ }^{26}$.e, MultiMate plasmid is shuttled into the MultiBac BV by Tn7-mediated recombination in E. Coli. MultiMate and MultiBac BVs can be further functionalised by in vitro Cre-mediated recombination (d-e). f, Plasmid size, number of modules and cargo to prokaryotic backbone DNA ratio (green and grey bars, respectively) for hypothetical $1.5 \mathrm{~kb}$ expression cassettes. g, Assembly strategy for MultiMate plasmid encoding chaperonin CCTs. CCT subunits were pasted into pMMK ENTR plasmid modules comprising baculoviral promoters (polH, p10), assembled by LR reaction into pMMACE DEST and pMMDS DEST, fused by Cre and loaded on EMBacY ${ }^{32}$ BV. Expression of MultiMate-CCTs with EMBacY in Sf21 insect cells produces complete CCT/TriC chaperonin. h, Western blot of infected Sf21 cells, uninfected cells as control (ctr) with CCT subunit specific antibodies. Anti-His was used to detect His-CCT $\alpha$. i, SDS-PAGE analysis of purified CCT/TriC chaperonin complex. j, Negative-stain EM (top) and 2D-class averages (bottom) of purified CCT/TriC. Scalebar, $100 \mathrm{~nm}$.

\section{Extended Data Figure 2. MultiMate assembly for baculovirus-vectored delivery of large}

multifunctional DNA in human cells. a, MultiMate-Rainbow assembly in a schematic view. Individual modules in pMMACE, pMMDS DEST and pMMK ENTR as shown (upper panel). Two LR reactions resulted in MultiMate-5-colours and pMMDS-2-colours (middle panel), fused by Cre-mediated recombination in vitro to produce MultiMate-Rainbow. b, Restriction mapping of five clones each of MultiMate-5-colours (left) pMMDS-2-colours (middle) and Cre-recombined MultiMate-Rainbow (right) evidences robust assembly. c, Confocal images of HEK293T, HeLa, H4 and SH-SY5Y cells 48 hours after transduction with MultiMate-5- 
691 colours VSV-G pseudotyped $\mathrm{BV}^{19}$. Scalebar, $20 \mu \mathrm{m}$. d, Schematic representation of

692 MultiMate-CellCycle assembly. Individual modules were pasted in pMMACE and pMMK

693 ENTR plasmids (upper panel). e, Restriction mapping of five clones each after LR

694 recombination. f, Confocal microscopy of HEK293T, SH-SY5Y, HeLa and H4 cells at 48

695 hours after transduction with MutiMate-CellCycle VSV-G pseudotyped BV. Scalebar, $100 \mu \mathrm{m}$.

696

697 Extended Data Figure 3. Baculovirus-vectored delivery of complete multicomponent

698 CRISPR/Cas9 toolkits for homology independent targeted integration (HITI) in human

699 cells, continued. a-c, HEK293T cells transfected with MultiMate-HDR or HITI-2c all-in-one

700 plasmids in the absence of puromycin selection at three- or 14-days post transfection. a,

701 representative flow-cytometry plots; $\mathbf{b}$, Histograms of absolute (left) and relative (right) gene

702 editing efficiencies. Flow-cytometry data of $\mathrm{n}=3$ independent biological replicates.

***P $<0.001$, Student's t-test. Relative gene editing efficiency is calculated as the $\%$ of

mCherry+ cells over the \% of eGFP+ cells. c, Confocal microscopy of HEK293T at two-days post transfection with MultiMate-HDR or MultiMate-HITI-2c plasmids. Scalebar, $50 \mu \mathrm{m}$. d,

Representative flow-cytometry histograms of HEK293T transfected with MultiMate-HDR or

MultiMate-HITI-2c after puromycin selection. e, Genotype PCR of HEK293T transfected (upper panel) or transduced (lower panel) with MultiMate-HDR or MultiMate-HITI-2c after puromycin selection. f, Widefield microscopy images of live HEK293T transfected with MultiMate-HDR or MultiMate-HITI-2c plasmids after puromycin selection. Scalebar, $100 \mu \mathrm{m}$.

711 g, Western blot of total protein extracts from puromycin selected HEK293T transfected with MultiMate-HDR, MultiMate-HITI-2c and untransfected HEK293T as a control (ctr). Anti- $\beta$ -

713 actin antibody was used in top panel, with anti-TUBULIN as a loading control. h,

714 Representative confocal images of HEK293T, HeLa, H4 and SH-SY5Y human cells 72 hours

715 after transduction with MultiMate-HDR or HITI-2c BV. Scalebar, $50 \mu \mathrm{m}$. i, Representative 
716 flow-cytometry plots of HEK293T, HeLa, H4 and SH-SY5Y cells at three-days post

717 transduction with MultiMate-HITI-2c BV, untransduced parental cell lines as a control (ctr).

Extended Data Figure 4. Baculovirus-vectored safe-harbour homology-independent

integration of large DNA cargoes in human genomes, continued. a-b, Delivery efficiencies

with the indicated MultiMate-HITI-2c plasmids. Mean \pm s.d. of $n=3$ independent biological

replicates. Dots and triangles represent total MultiMate plasmid and HITI-2c payload DNA

sizes, respectively. e-f, Representative flow-cytometry histograms of HEK293T transduced

with the indicated MultiMate-HITI-2c BVs and selected with puromycin or

puromycin/hygromycin for mCherry expression (e) or eGFP/EYFP expression (f).

730 Extended Data Figure 5. Highly efficient and indels-free prime-editing by using

MultiMate all-in-one BV, continued a, Restriction mapping of five randomly picked

MultiMate-PE2 clones. b, Confocal microscopy images of HEK293T two-days after of HEK293T four-days after transfection with MultiMate-PE2 and relative sequences contribution after Sanger sequencing deconvolution (ICE). Mean \pm s.d. of $n=3$ independent biological replicates. d, Visual summary of BV amplification. V0: widefield images of Sf21 cells in 6-well plates after initial transfection with MultiMate-PE2 BV DNA and control cells (untransfected). All transfected cells show VSV-G induced syncytia. V2: aeBlue expression in suspension culture (upper panel) and centrifuged pellets (lower panel) of Sf21 cells during amplification of MultiMate-PE2 BVs. e, Confocal microscopy images of HeLa, RPE-1 
741 hTERT, HEK293T and SH-SY5Y at 24-hours post transduction with MultiMate-PE2 BV. f,

742 Representative flow-cytometry histograms of mTagBFP expression in HEK293T at 24-hours

743 post transduction. Dotted lines represent gating. g, Transduction efficiencies of HEK293T at

744 24-hours post-transduction with the indicated dilutions of MultiMate-PE2 BV. Mean \pm s.d. of

$745 \mathrm{n}=5$ independent biological replicates. $\mathbf{h}$, Sanger sequencing comparisons of of HEK3 locus

746 in HEK293T six days after transduction with the indicated amounts of MultiMate-PE2 BV. i,

747 Percentage of correct editing (CTT insertion) in HEK293T six days post transduction with the

748 indicated amounts of MultiMate-PE2 BV. Data are derived from Sanger sequencing

749 deconvolution (ICE), no indels were detected in any of the conditions. Mean \pm s.d. of $n=3$

750 independent biological replicates. 

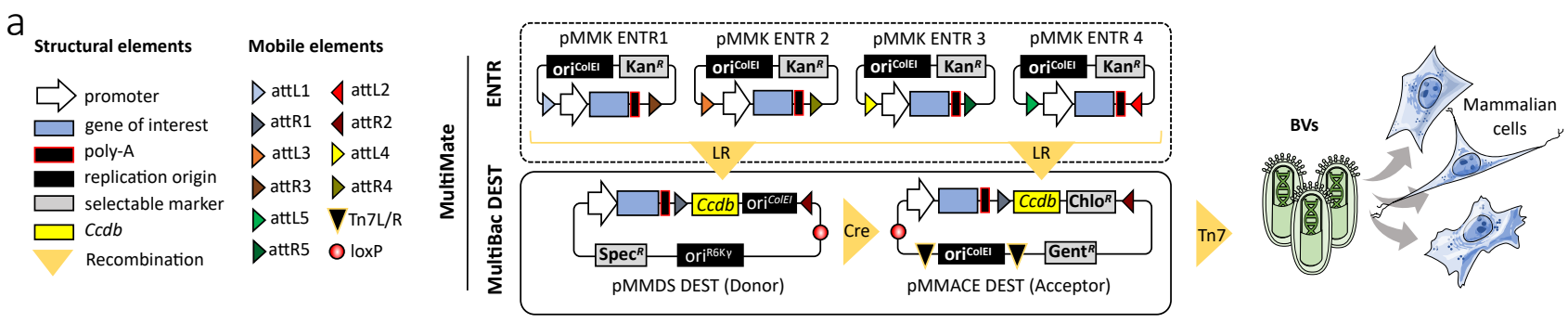

b

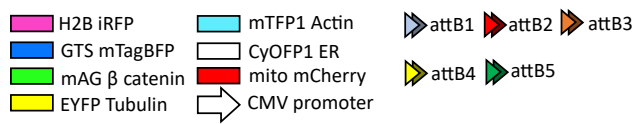

(2) In7 (23.4 kb)

C
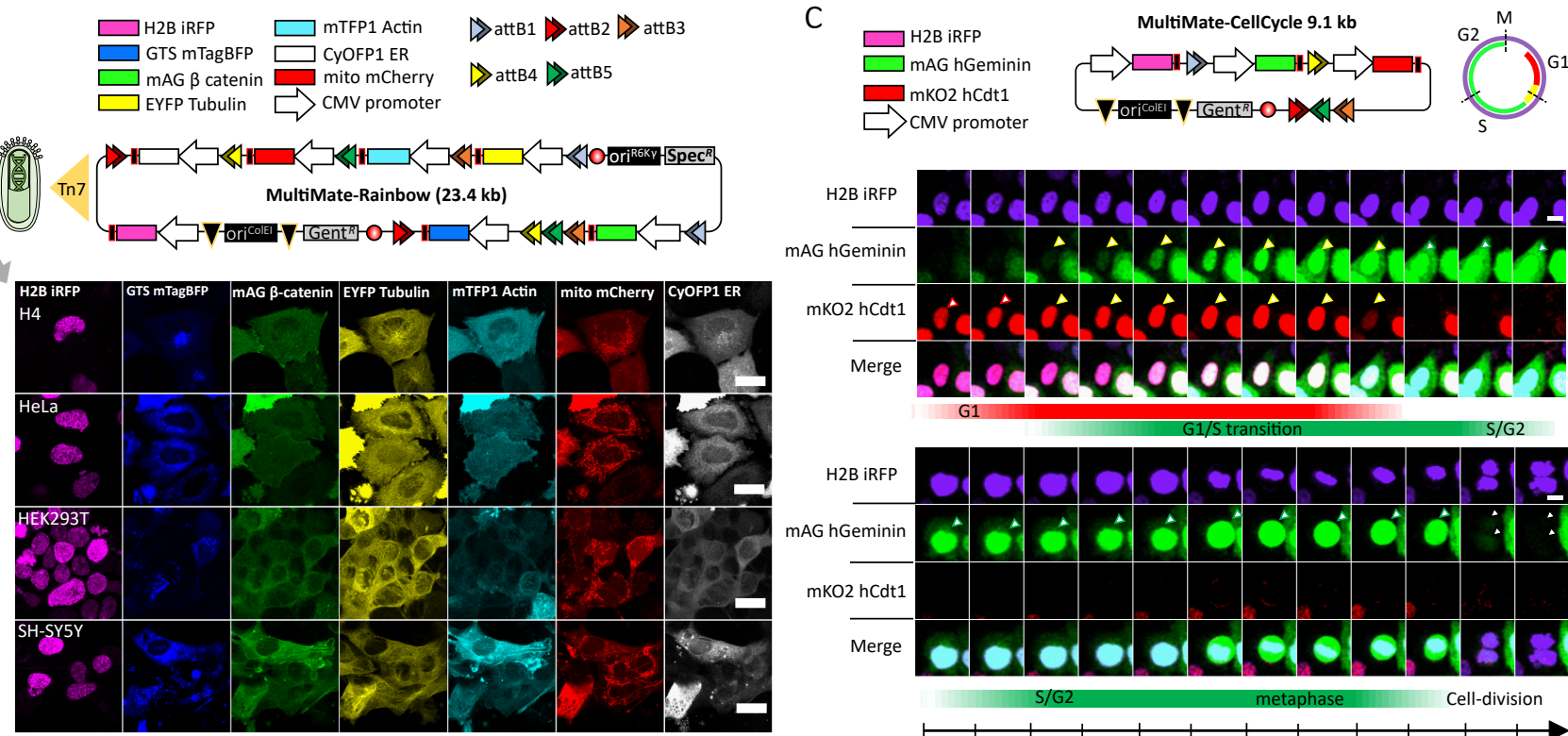

mAG hGeminin mKO2 hCdt1

Merge

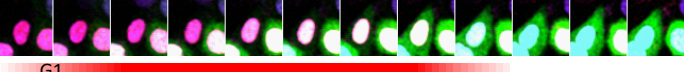
$\begin{array}{lll}\text { G1 G1/S transition } & \text { S/G2 }\end{array}$
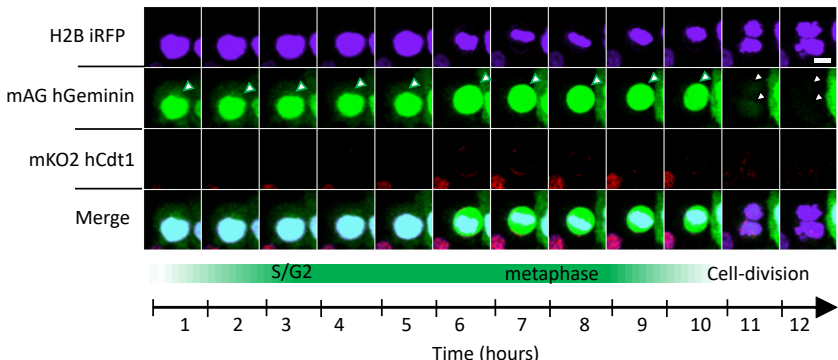

Fig1_AulicinoBerger 

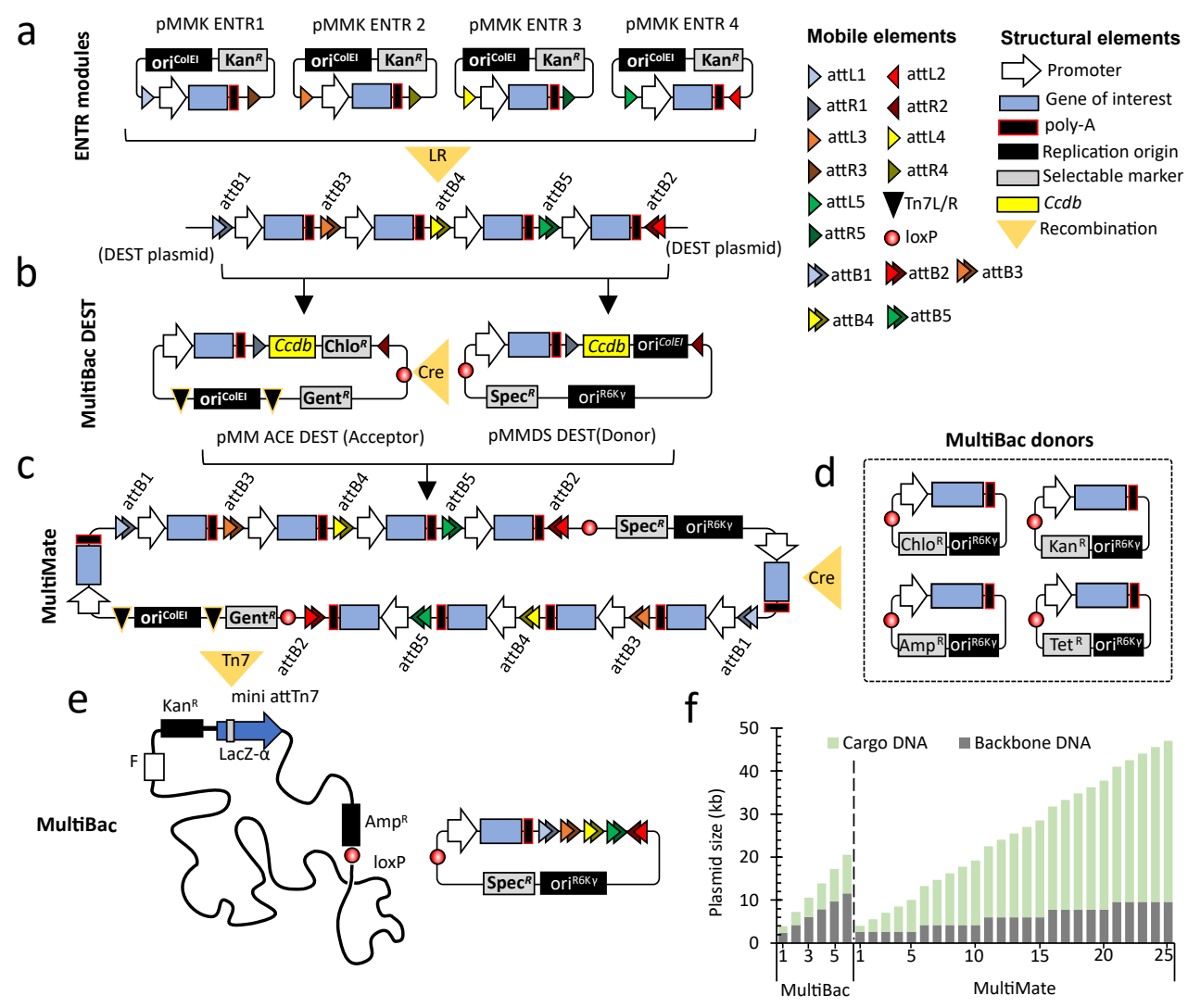

Number of DNA modules

$\mathrm{g}$

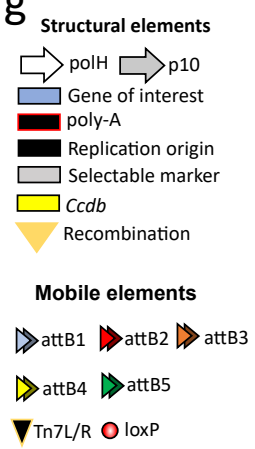

DEST PMMACE DEST

ENTR 1 PMMK ENTR 1 polH CCT ENTR 2 PMMK ENTR 2 p10 CCTY

ENTR 3 PMMK ENTR 3 polH CCTB

ENTR 4 PMMK ENTR 4 p10 CCT $\delta$

LR

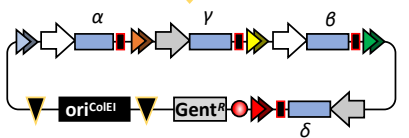

CRE
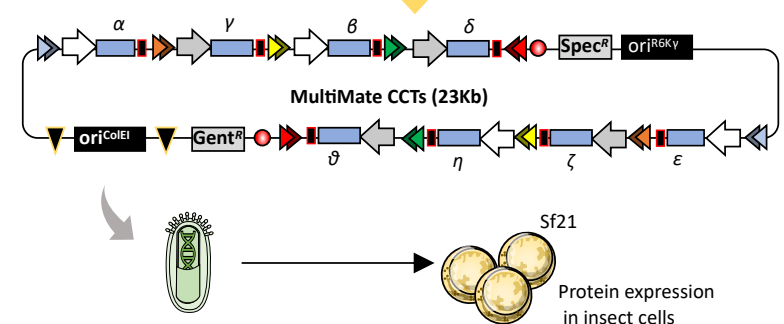

DMMDS DEST

ENTR 1 PMMK ENTR 1 polH CCT $\varepsilon$

ENTR 2 PMMK ENTR 2 p10 CCTS

ENTR 3 pMMK ENTR 3 polH CCT $\eta$

ENTR 4 PMMK ENTR 4 p10 CCT $\vartheta$

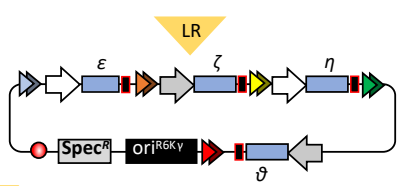

CRE

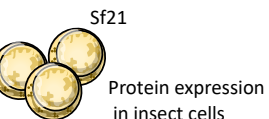

h
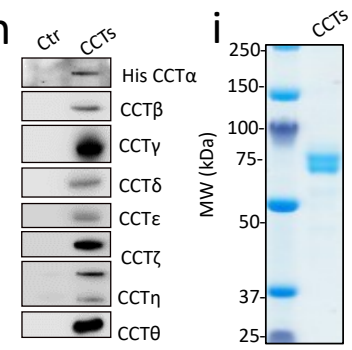

j

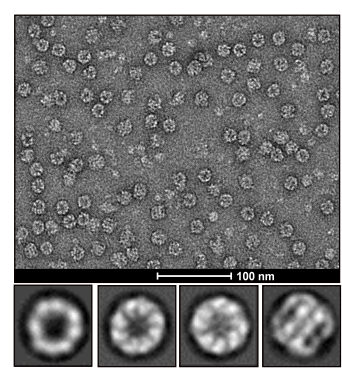

\section{ExtDataFig1_AulicinoBerger}


DEST PMMACE DEST CMV H2B

ENTR 1 pMMK ENTR 1 CMV EYFP Tubulin $\square$ ENTR 1 pMMK ENTR 1 CMV mAG $\beta$-catenin $\square$

ENTR 2 PMMK ENTR 2 CMV mTFP1 Actin

ENTR 3 PMMK ENTR 3 CMV mito mCherry

ENTR 4 PMMK ENTR 4 CMV CYOFP1 ER

ENTR 2 PMMK ENTR 2 Empty vector

ENTR 3 PMMK ENTR 3 Empty vector

ENTR 4 PMMK ENTR 4 CMV GTS mTagBFP $\square$

$$
\text { LR }
$$

LR
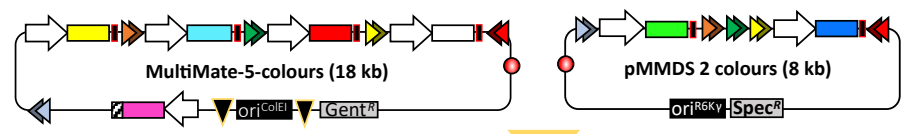

Cre
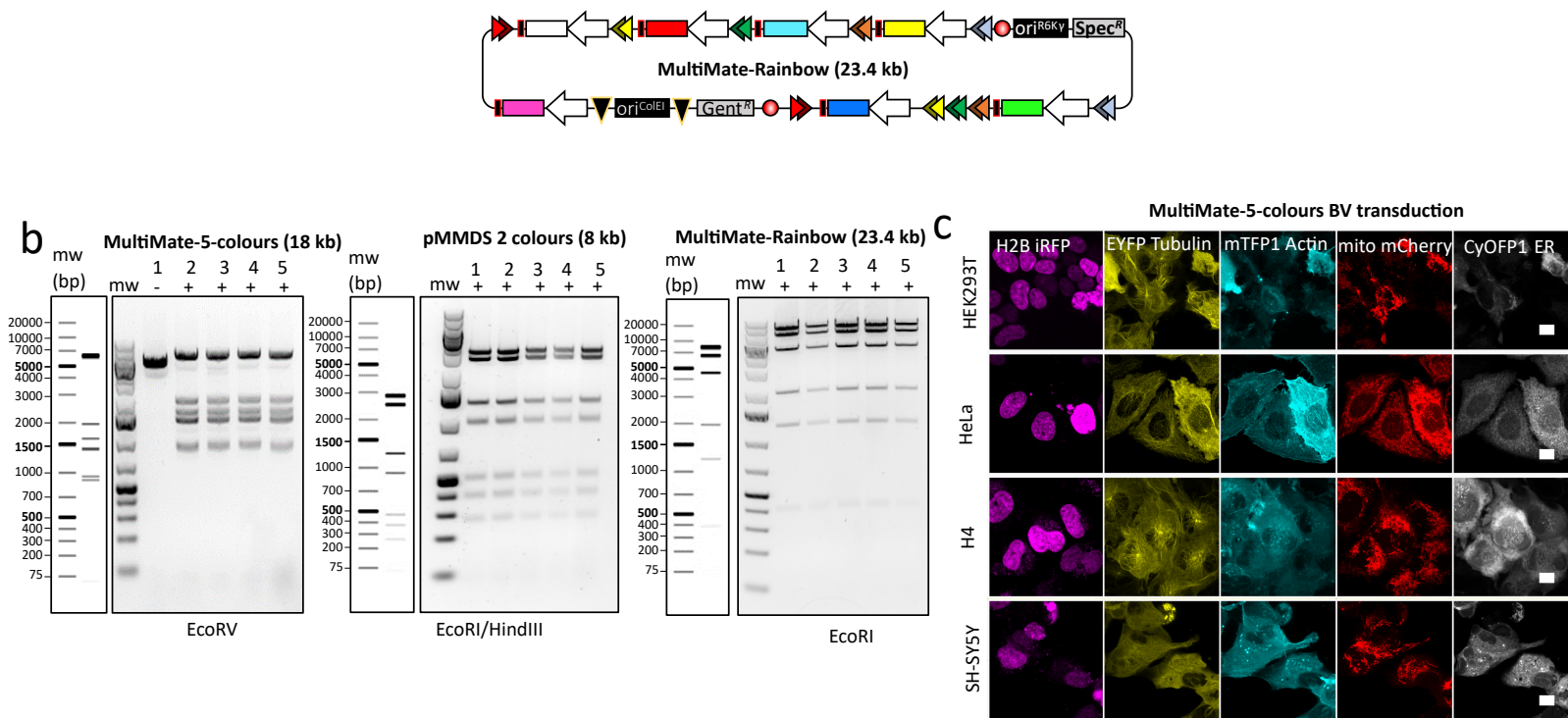

d

$\begin{array}{ll}\text { DEST } & \text { pMMACE DEST CMV H2B-iRFP } \\ \text { ENTR } 1 & \text { pMMK ENTR1 CMV mAG-hGeminin }\end{array}$ ENTR 2 pMMKENTR2 CMV mKO2-hCdt1

ENTR 3 PMMK ENTR 3 empty vector

ENTR 4 PMMK ENTR 4 empty vector

MultiMate-CellCycle (9.1 kb)

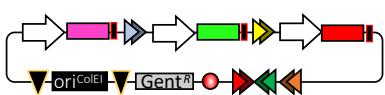

e

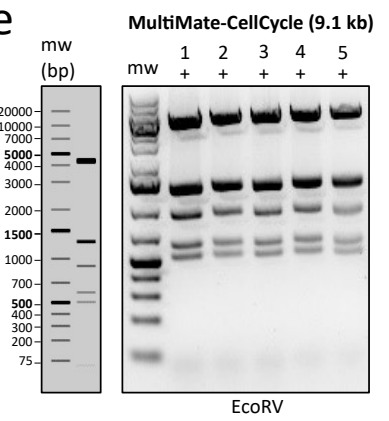

$f$

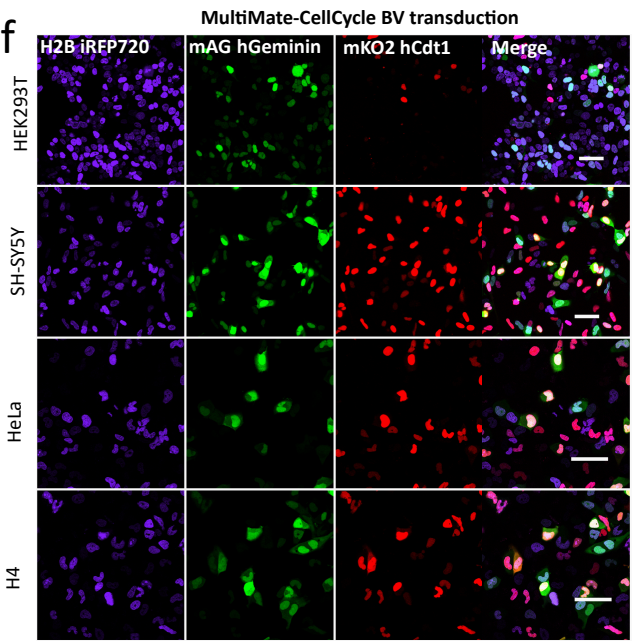

\section{ExtDataFig2_AulicinoBerger}


a Promoters

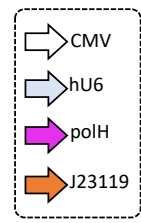

\section{$\square$ SpCas9}

$\square$ eGFP

Acrll4

$\square$ Target

$\square$ sgRNA

$\square$ Donor

$\square$ Exon

d

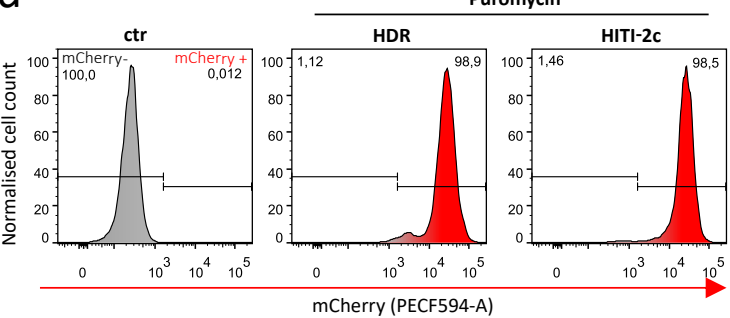

e

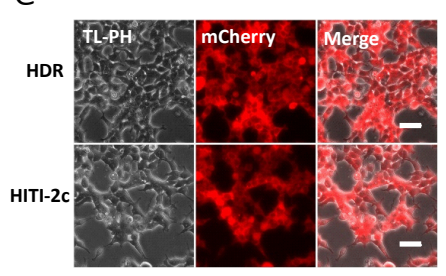

Fig2_AulicinoBerger

$f$

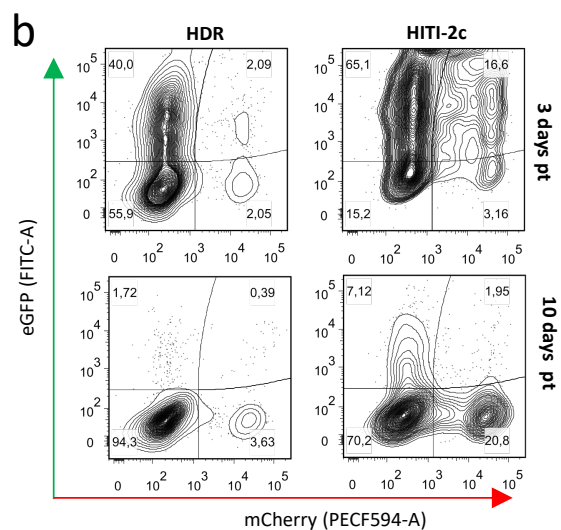

C

mCherry+

- eGFP+ mCherry+

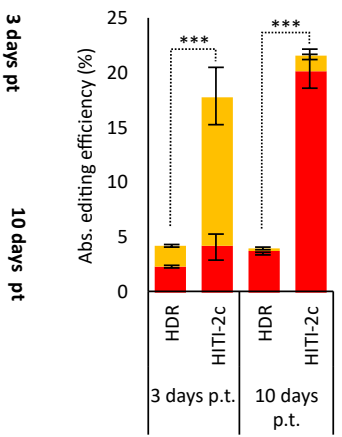

g
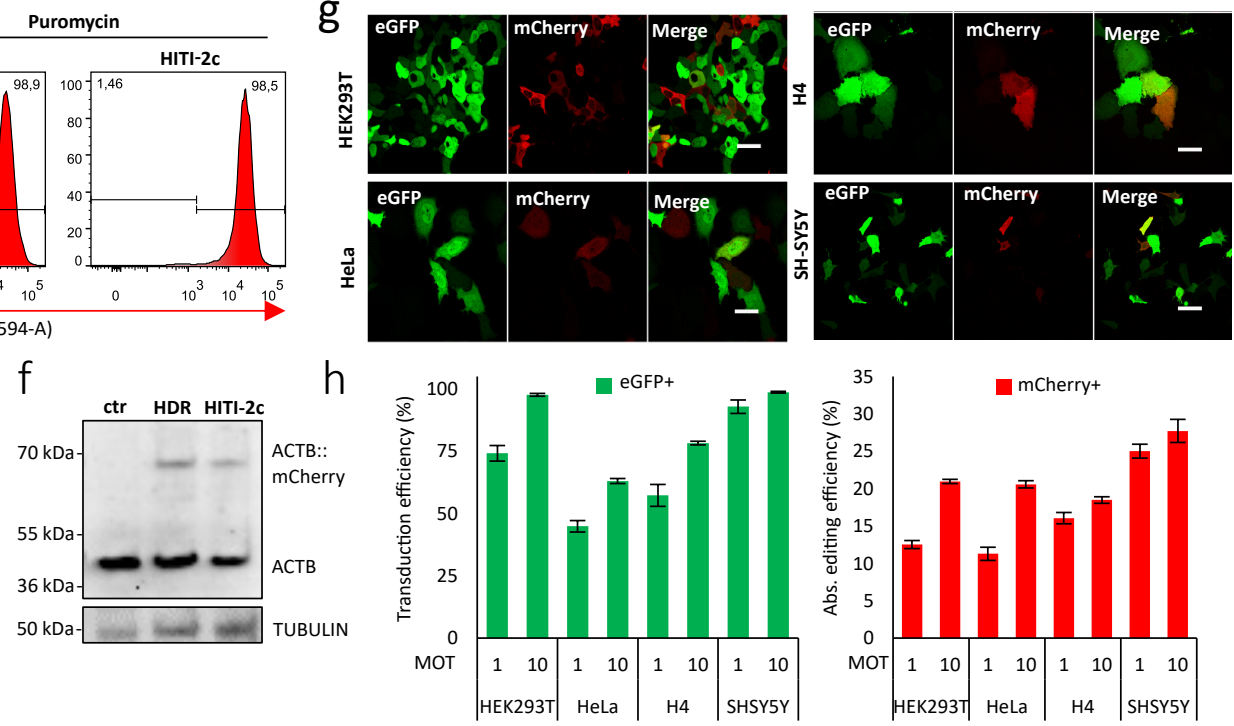
bioRxiv preprint doi: https://doi.org/10.1101/2020 08.17.253898; this version posted August 17, 2020. The copyright holder for this preprint (which was not certified by peer review) is the author/funder, who has granted bioRxiv a license to display the preprint in perpetuity. It is made available under aCC-BY-NC-ND 4.0 International license.
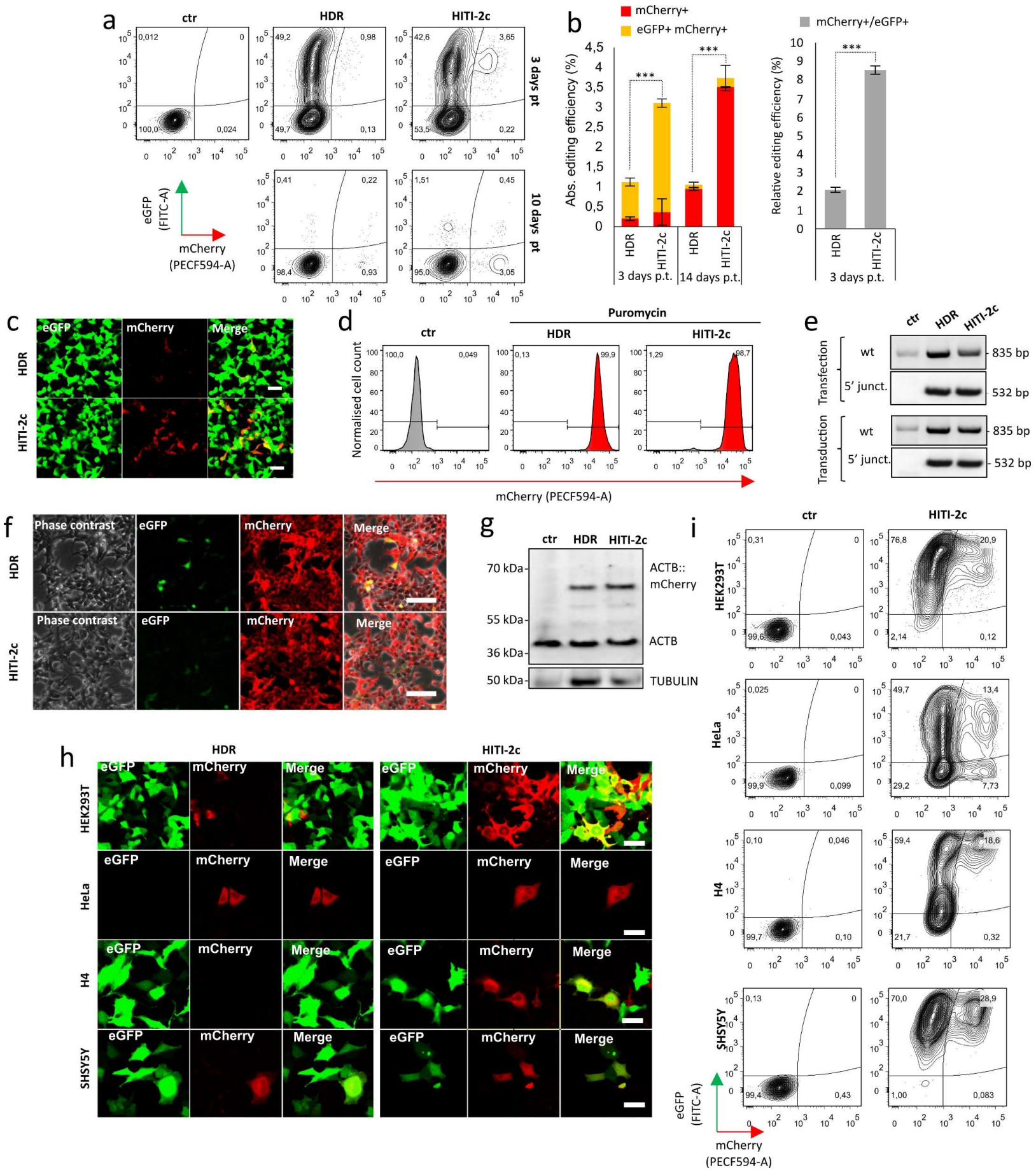

ExtDataFig3_AulicinoBerger 
Exons

f

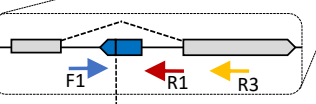

$\square$ target

HITI-2c expansion payloads

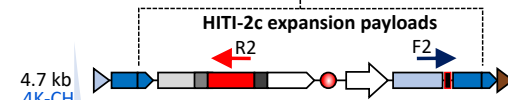

$\neg$ hEF1a

$6 \mathrm{~kb}$

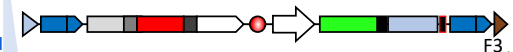

$\square$ Puro-R

TT2A IRES

P2A O loxp

$\square$ Hygro-R

$\square$ eGFP

$\square$ EYFP Tubulin

$9 \mathrm{~kb}$

$9 \mathrm{~kb}$
$9 \mathrm{~K}-\mathrm{EYH}$

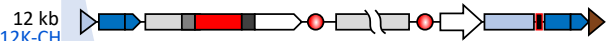

$\square \square$ Cargo

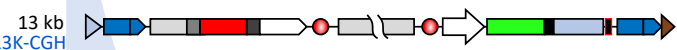

$17 \mathrm{~kb}$
$1 \mathrm{~K}-\mathrm{EYH}$

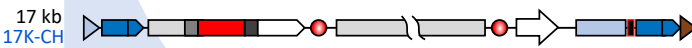

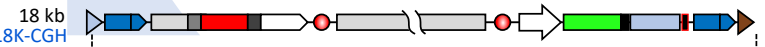

HITI-2c payload

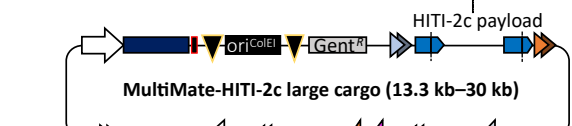

d

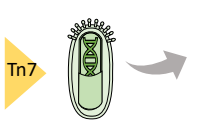

C

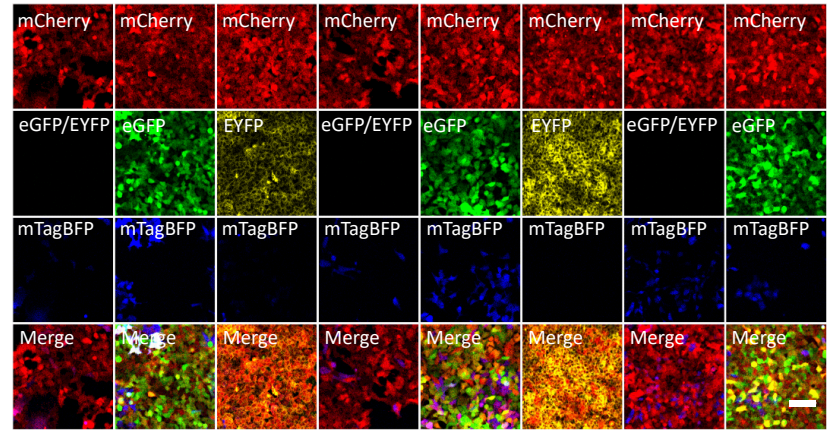

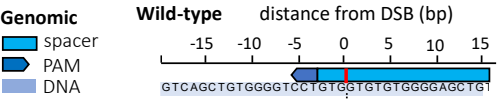

Donor

$\square$ spacer

$\square$ PAM

$18 \mathrm{~K}-\mathrm{CGH}$

$5^{\prime}$ junction (pool)

nction (pool)

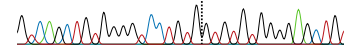

$\begin{array}{lllllll}-15 & -10 & -5 & 9 & 5 & 10 & 15\end{array}$

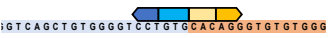

STCAGCTGTGGGTCCTGTGCACAGGGTGGTGG

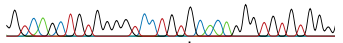

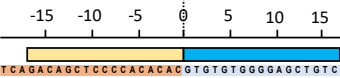

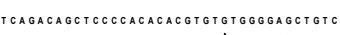

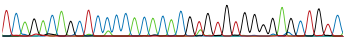

e

ஓ $\cong$ mTagBFP+

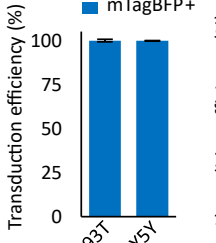

f

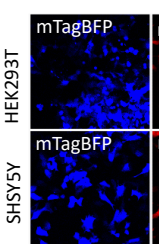

C

$3^{\prime}$ junction

(CMV payloads)

$3^{\prime}$ junction

(hEF1 $\alpha$ payloads
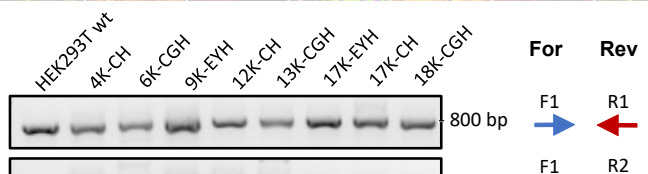

$\stackrel{\mathrm{F} 1}{\mathrm{R2}}$

F2 R3

$\rightarrow<-$

$-\quad 438 \mathrm{bp} \stackrel{\mathrm{F} 3}{\rightarrow} \mathrm{R}^{\mathrm{R} 3}$

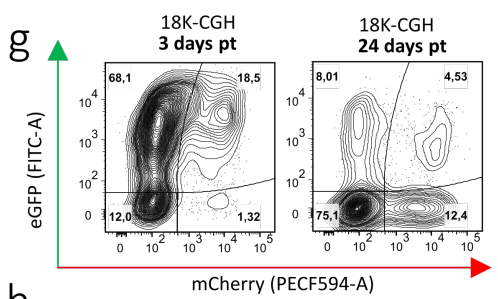

h
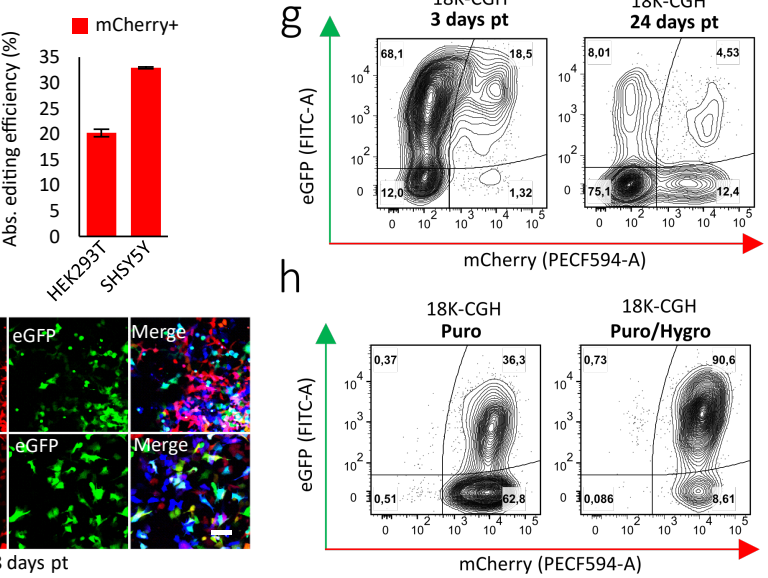

Fig3_AulicinoBerger 
Transfection

a

- mTagBFP+ Plasmid size $\triangle$ HITI-2c payload size

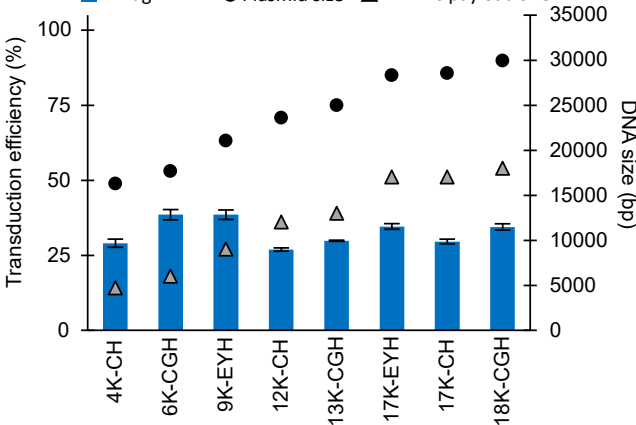

C

Transfection
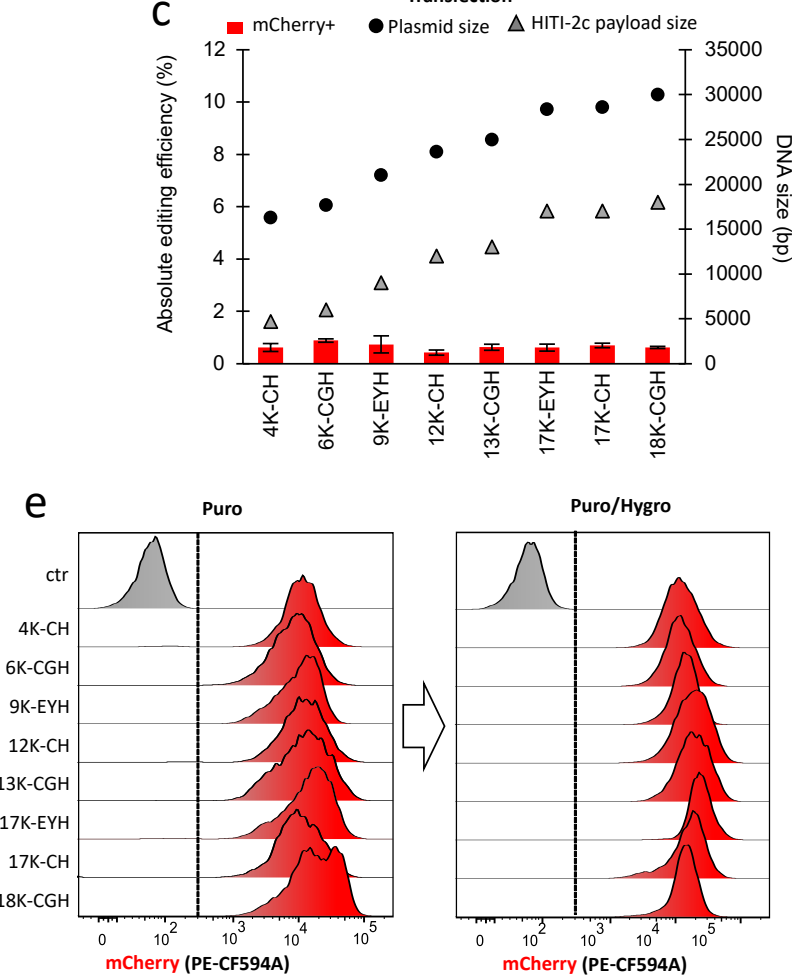

b EMBacY Transduction (MOT $<10$ )

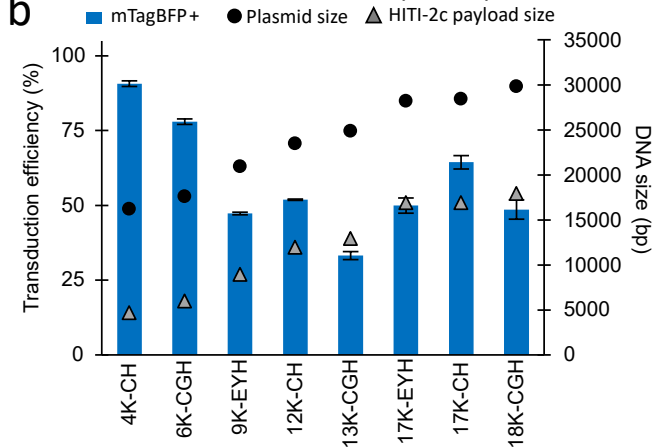

EMBacY Transduction (MOT <10)

d $\quad$ mCherry+ $\quad$ Plasmid size $\triangle$ HITI-2c payload size

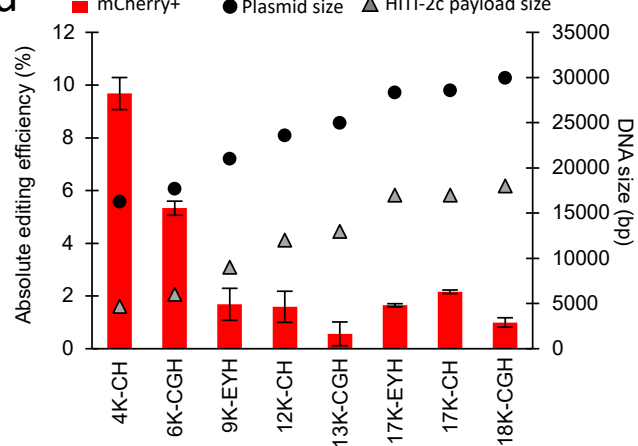

f
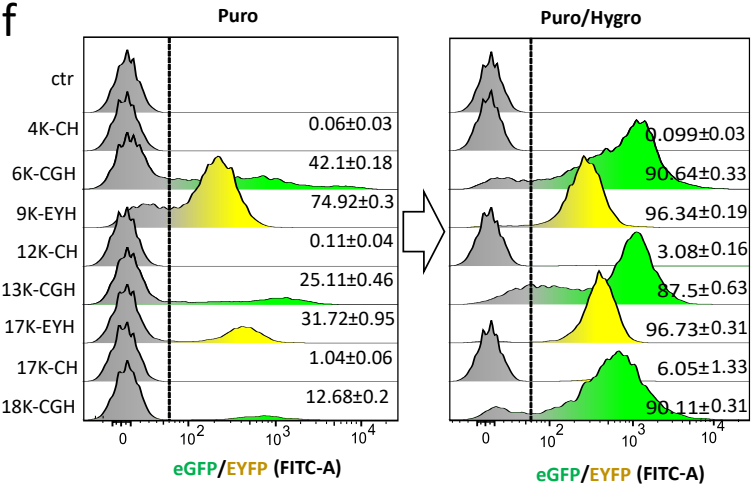

ExtDataFig4_AulicinoBerger 
a

\begin{tabular}{|c|c|c|}
\hline$\square$ & $\square$ PE2 & $\square_{\text {VSV-G }}$ \\
\hline & $\square$ PegRNA & $\square$ mTagBFP \\
\hline & aeBlue & $\Longrightarrow$ Poly-A \\
\hline
\end{tabular}

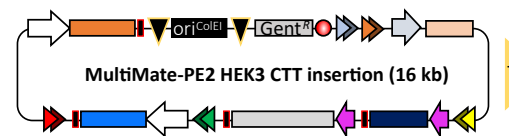

d

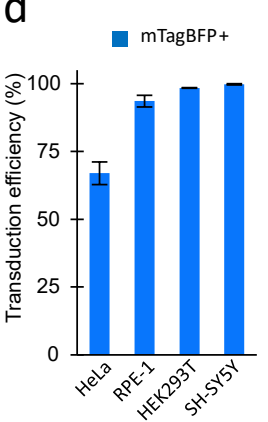

Fig4_AulicinoBerger

\section{e}

CTT insertion
(wo indels)

b

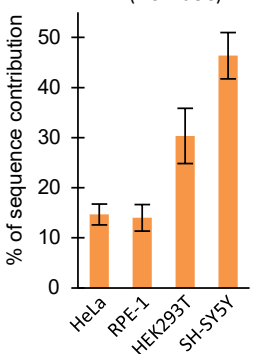

Tn7

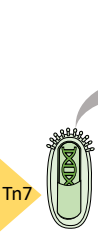

迹

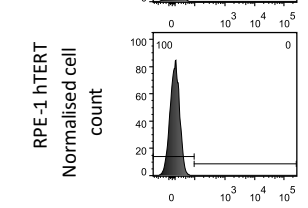

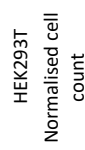

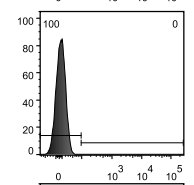

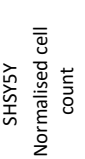
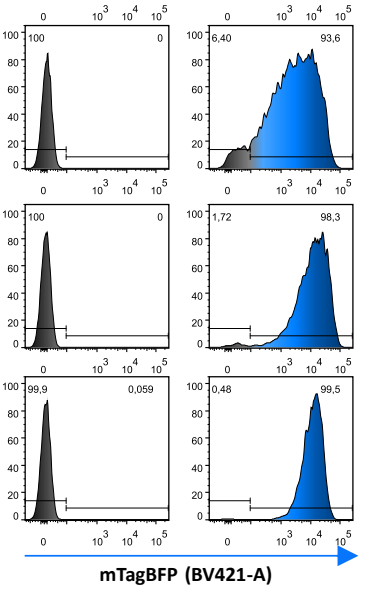

C CTT insertion

PegRNA target

Hela PAM

GCACGTGATGGCAAAGGAAAGGAGCCCTC

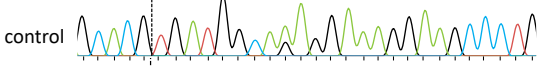

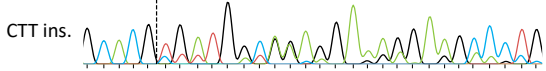

RPE1-hTERT GCACGTGAT GGCA GAGGAAGGAAGCC T

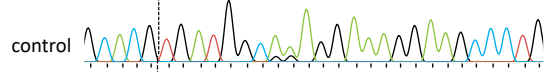

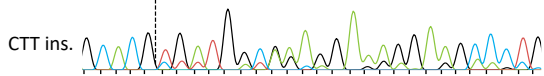
HEK293T G CAC G T G AT G GCA G A G G A A A G G AA GC C C T G

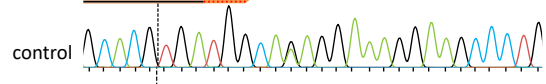

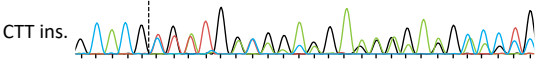
SHSY5Y GCACGTGATGGCAGAGGAAAGGAGCCCTG

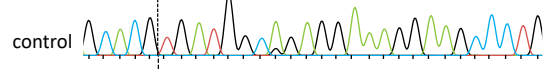
Distance from nicked site (bp) 
a

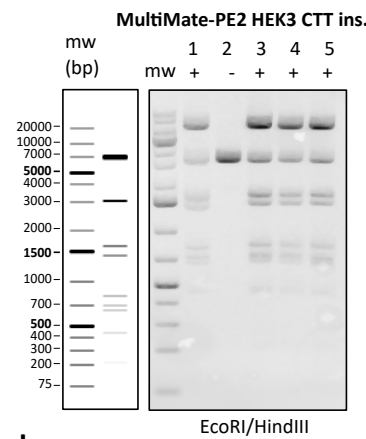

d

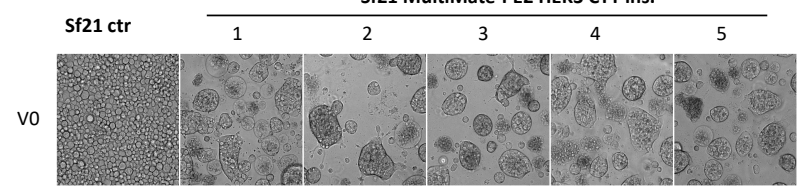

V2

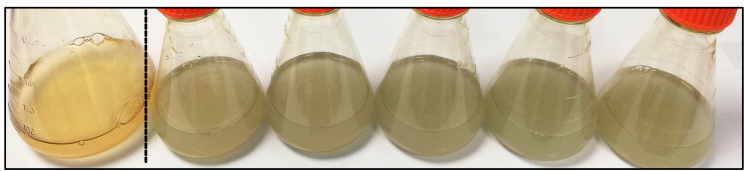

aeBlue+ cell pellets

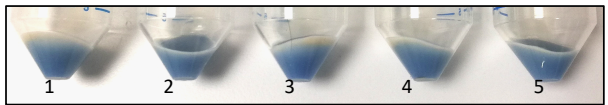

C PegRNA target

Plasmid transfection

MultiMate-PE2 HEK3 CTT ins. 48 hrs p.t.

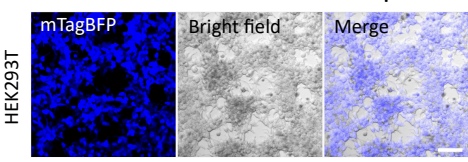

GGCCCAGACTGAGCACG:T G AT G GCA G A G A AA G G

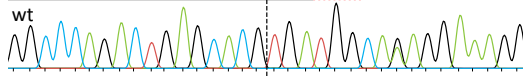
$\underset{-15}{\text { ME2 CTT }} \underset{-10}{\text { Distance from nicked site (bp) }}$

Sanger sequencing deconvolution (ICE) Contributions wt GGGCCCAGACTGAGCACG TGATGGCAG $86 \pm 0 \%$ CTT ins. GGGCCCAGACTGAGCACG CTTTGATGG $13 \pm 0 \%$

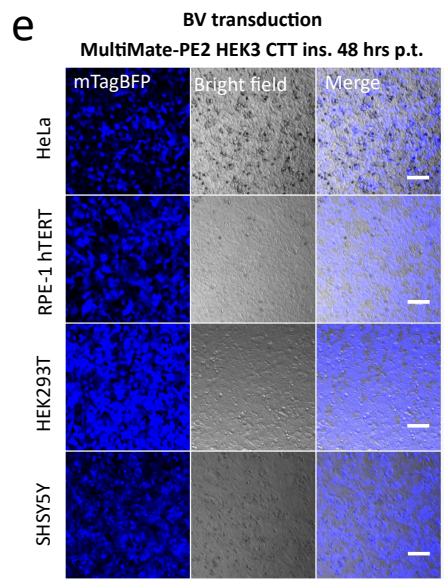

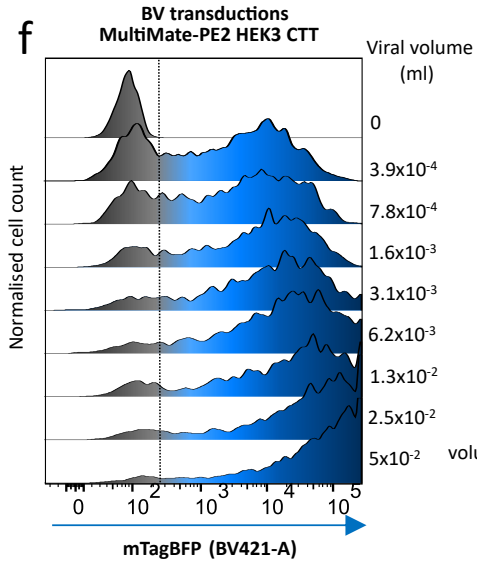

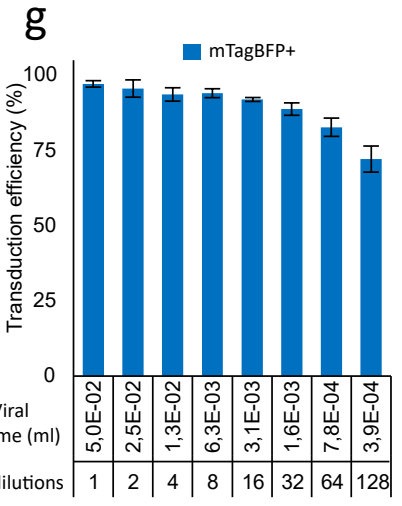

h PegRNA target $\longrightarrow$ PAM

wt TGAGCACGTGATGGCAGAGGA cTT ins. TGAGCACGITGATGGCAGAGGA ${ }_{\&}$ Control MhWM Mh M M

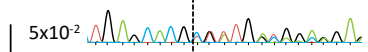

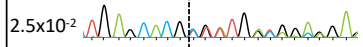

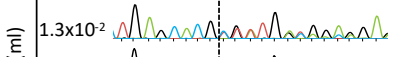
है $6.2 \times 10^{-3}$ Mhmon

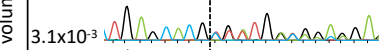

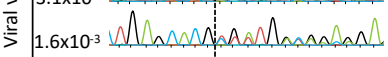

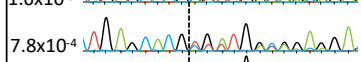
$3.9 \times 10^{-4} \mathrm{~m}$ M M M A ham

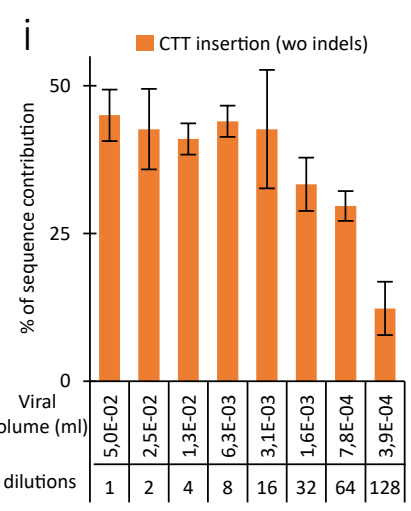

\section{ExtDataFig5_AulicinoBerger}

